\title{
États et propriétés des déclinaisons arabes
}

Par

\section{Pierre Hanjoul}

Université catholique de Louvain, Louvain-la-Neuve

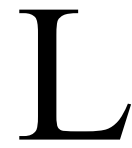

a notion d'état est apparue avec l'état construit, dans les travaux de grammairiens chrétiens de l'hébreu biblique des $\mathrm{XVI}^{\mathrm{e}}$ et $\mathrm{XVII}^{\mathrm{e}}$ siècles ${ }^{1}$; de là, elle a été étendue à d'autres états et à d'autres langues. Mais alors qu'elle est assez bien établie dans l'étude des langues sémitiques en général, elle reste peu utilisée à propos de l'arabe, étant même absente du Traité de philologie arabe (FLEISCH 1956) et, comme nous le verrons plus loin, au sens qui nous concerne, de l'Encyclopedia of Arabic Language and Linguistics (Versteegh 2005-2008). Pourtant, ma pratique de l'enseignement de l'arabe m'a convaincu qu'elle constitue la meilleure approche des déclinaisons de cette langue. Elle en permet une description claire, précise et concise et simplifie l'énoncé de certaines règles grammaticales ; tous, tant enseignants et étudiants que chercheurs, gagneraient à l'utiliser. Il me paraît donc important de la faire mieux connaître.

Une autre raison d'écrire cet article est que certains manuels et grammaires de l'arabe utilisent de multiples expressions telles que « défini par la forme », « de sens déterminé », etc., et ce d'une façon parfois confuse, voire incohérente. Il m'a semblé bon de mettre de l'ordre dans ce vocabulaire sur la base de l'usage linguistique actuel; or cette question touche, comme on va le voir, aux états. Une troisième raison, enfin, est née de ce travail lui-même. Étudiant les états, j'ai été amené à me poser la question de leur définition exacte et de leur relation aux cas, et à explorer quelques recoins méconnus de la grammaire arabe pour vérifier si le sys-

\footnotetext{
${ }^{1}$ Voir P. Van Hecke, Construct State: Biblical Hebrew, p. 579, dans l'Encyclopedia of Hebrew language and linguistics (KHAN 2013).
} 
tème des cas et états y restait pertinent. Les résultats de cette recherche m'ont paru suffisamment intéressants pour justifier par eux-mêmes une publication.

Notre présentation se fera en deux parties. Dans la première, nous étudierons comment peut s'élaborer logiquement la notion d'état en arabe. Décrire ce processus, établir la cohérence de la notion et dégager progressivement une définition et une perspective claires m'a paru utile étant donné sa faible diffusion auprès des arabisants ; en outre, à en croire RETSÖ 1997, p. 268-269, en particulier les n. 2, 3 et 5, les états, lorsqu'on en fait usage à propos d'autres langues sémitiques, ne sont pas toujours définis de façon claire. Nous verrons comment se présentent les tableaux de déclinaison quand on y inclut les états ; organisés de manière adéquate, ces tableaux font mieux prendre conscience de certaines propriétés des déclinaisons arabes, qui se traduisent par des propriétés graphiques des tableaux. Nous ferons tout cela en utilisant directement le vocabulaire qui nous semble le plus approprié, plutôt que de mentionner tout de go les diverses appellations qui apparaissent dans la littérature grammaticale. La terminologie est en effet loin d'être unifiée, et nous terminerons cette première partie par une revue de celle-ci. Le fait que la notion d'état soit peu utilisée à propos de l'arabe est peut-être une chance : elle nous donne la possibilité de proposer un vocabulaire mieux adapté sans pour autant devoir bousculer une tradition vraiment établie.

Nous réservons à la seconde partie certains problèmes et propriétés qui montrent que, pour ce qui est de l'arabe, la cohérence du système des cas et états s'étend plus loin qu'on ne le conçoit ordinairement.

\section{Perspective générale}

\section{États construit / inconstruit}

L'état construit (status constructus) est commun à l'ensemble des langues sémitiques. Pour ce qui est de l'arabe, en première approximation - nous reviendrons sur ce point dans la seconde partie -, l'état construit désigne, pour un nom ${ }^{2}$ ou un adjectif (qualificatif, puisqu'il s'agit des seuls adjectifs de cette langue), l'ensemble des formes prises par ce mot lorsqu'il a un complément en annexion, c'est-à-dire un complément au génitif non introduit par une pré-

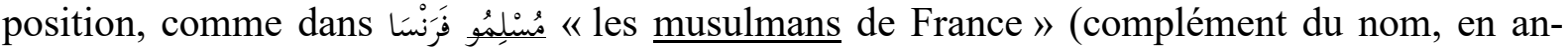
nexion réelle - ce complément pouvant être un nom, un pronom, ou encore une proposition

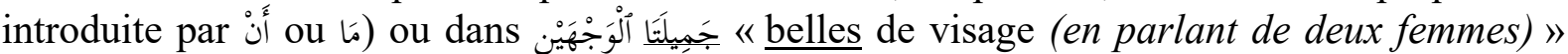
(complément de l'adjectif, en annexion formelle). Dans cet état, les pluriels masculins ex-

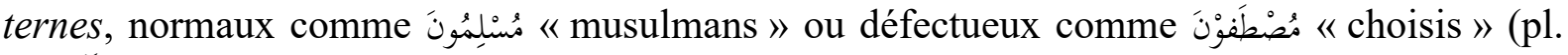

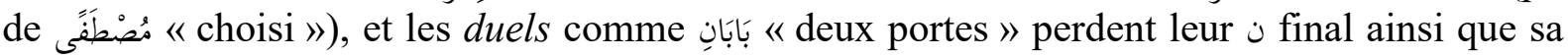
voyelle. Nous proposons d'appeler état inconstruit l'état contraire, c'est-à-dire la forme de ces mots avec leur u final ; cette dénomination, logique, souligne le parallèle avec les états déterminé et indéterminé des déclinaisons triptotes et apparentées dont nous parlerons plus loin.

La déclinaison des pluriels masculins externes et des duels peut donc être présentée dans des tableaux à trois lignes associées aux trois cas, et deux colonnes associées aux deux états. Dans tous les tableaux qui suivent, nous avons choisi de placer dans la case du haut à gauche la forme du mot tel qu'il est recensé dans la plupart des dictionnaires actuels : nominatif inconstruit pour les mots qui nous occupent ici, et, comme nous allons le voir, nominatif indéterminé pour les triptotes, diptotes et pluriels féminins externes. En outre, il nous a paru inté-

\footnotetext{
2 Catégorie dans laquelle nous pouvons inclure أَ أَيّْ 《quel, lequel» et ses diverses formes de genre et de nombre.
} 
ressant d'effacer les côtés séparant des cases contenant la même forme. Ceci fait apparaître des plages d'un seul tenant, dont chacune est associée à une seule forme.

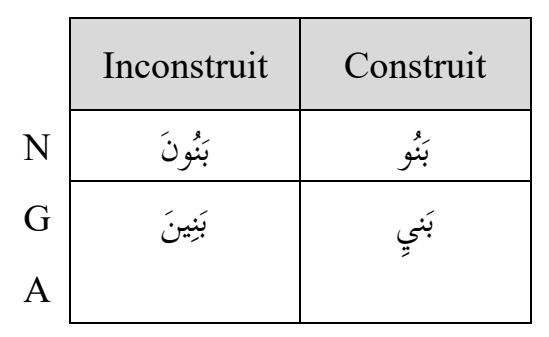

Pluriels masculins externes normaux: بَ بَنُونَ fils (pl.)»

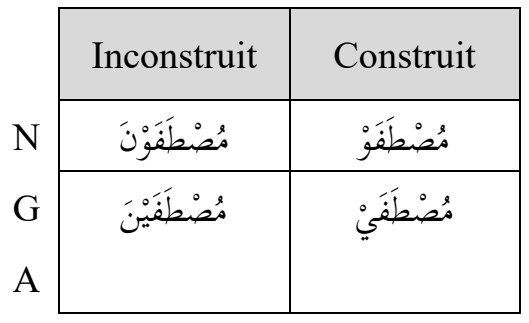

Pluriels masculins externes en -wna (défectueux) :

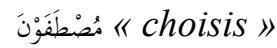

\section{États déterminé / indéterminé}

Moins familier que l'état construit, l'état indéterminé (status indeterminatus) semble, dans le cas des triptotes et des pluriels féminins externes, désigner quasi unanimement, pour l'arabe, les formes avec nunation. De même que dans le cas précédent, on peut donc présenter la déclinaison de ces mots (sauf pour les Cinq Noms, cf. infra) en tableaux dont les colonnes sont associées l'une à l'état indéterminé, l'autre à son contraire, que nous dirons, logique-

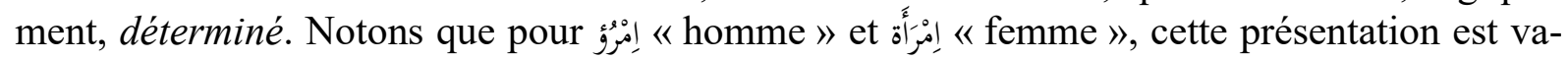
lable pour les désinences seulement : la forme de ces deux mots dépend aussi de la présence éventuelle de l'article; nous y reviendrons dans la seconde partie.

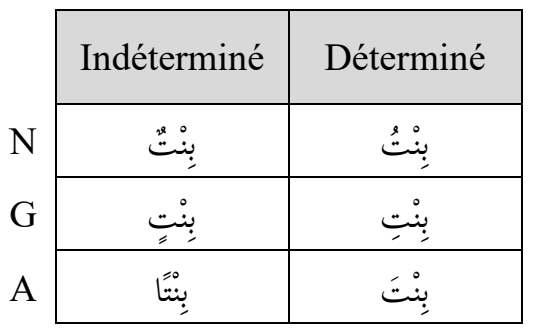

Triptotes normaux : بنْن « fille»

\begin{tabular}{|c|}
\hline Déterminé \\
\hline بَنَاتُ \\
\hline بَنَاتِ \\
\hline
\end{tabular}

Pluriels féminins externes: بَّنَ

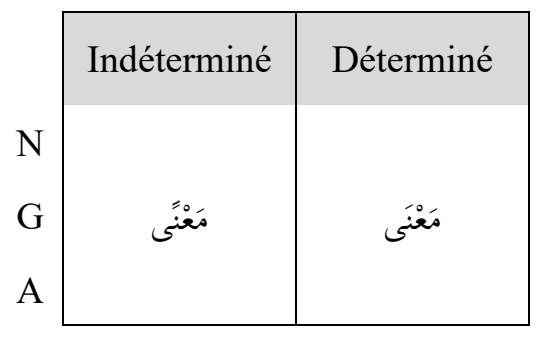

Triptotes en -an

(défectueux) :

هَ "signification 》

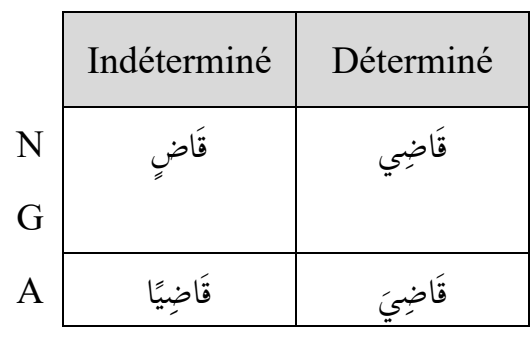

Triptotes en -in

(défectueux) :

قَاضٍ «juge » 
Pour ce qui est des diptotes, les formes ne peuvent guère être classées sur base de la présence ou absence de nunation, qu'il s'agisse des diptotes normaux comme آخَرُ "autre (masc.) 》, des diptotes en -â (indéclinables) comme أخخرَى 《autre (fém.) 》 ou même, partielle-

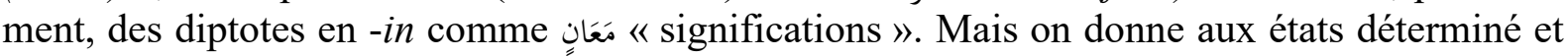
indéterminé une portée générale en les redéfinissant ainsi :

l'état déterminé (resp. indéterminé) est la réunion des situations où les triptotes normaux perdent (resp. conservent) leur nunation.

Ceci nous permet d'établir les tableaux, donnés ci-dessous, des déclinaisons diptotes normale et diptote en -in. Cette opposition déterminé / indéterminé doit en effet fonctionner pour les diptotes : on sait bien que ceux-ci se déclinent comme les triptotes dans les situations où ces derniers perdent leur nunation, et ont leur déclinaison particulière dans celles où les triptotes gardent leur nunation (chose que la grammaire traditionnelle exprime en disant qu'il n'y a de déclinaison diptote qu'à l'indéterminé). Ceci n'est pourtant pas tout à fait vrai, comme nous le verrons dans la seconde partie de cet exposé, mais contentons-nous en pour le moment.
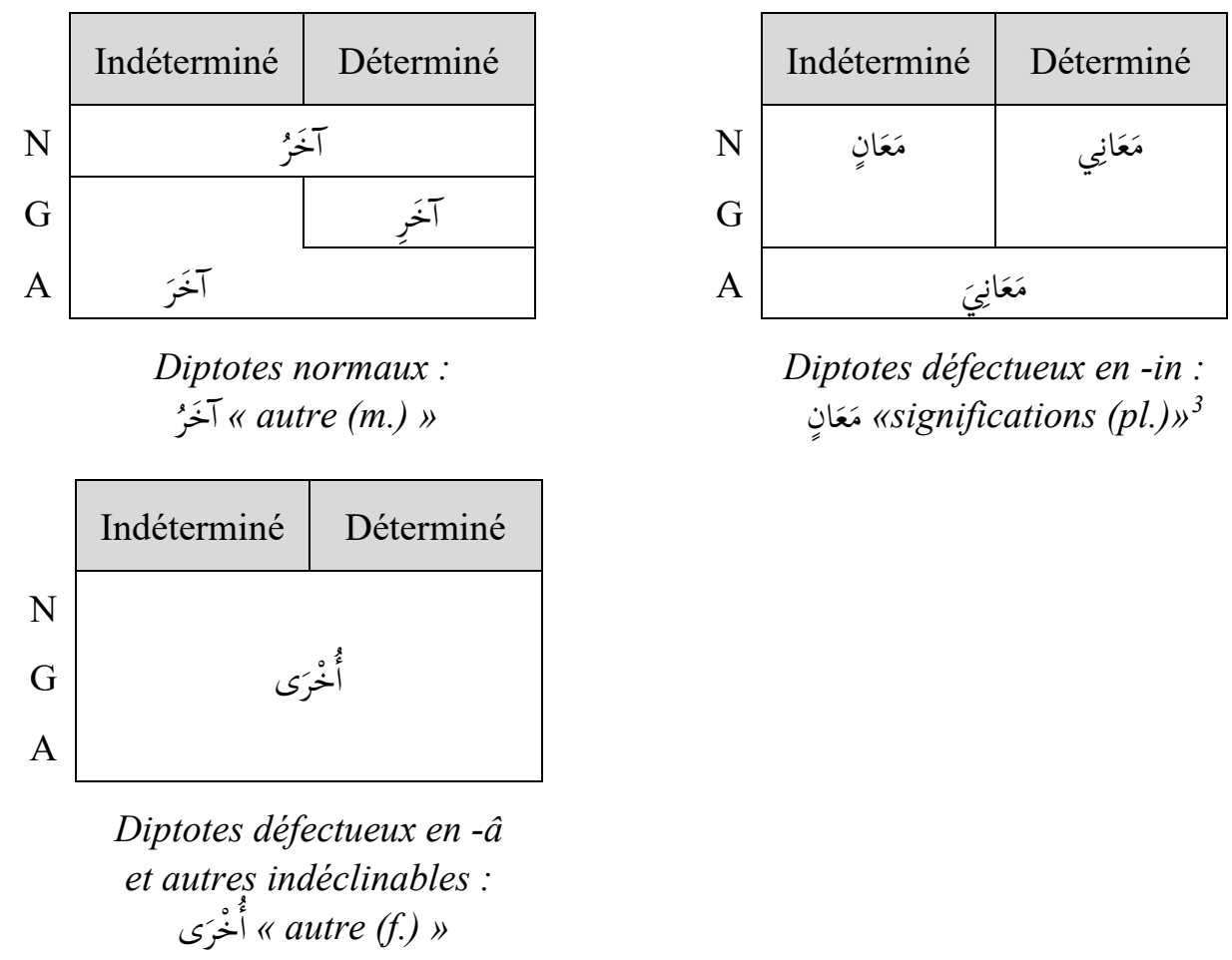

\section{Les Cinq Noms}

Cette manière d'envisager les états de l'arabe convient manifestement très bien à la présentation des déclinaisons de cette langue; elle est adoptée, avec une autre terminologie, par BISHAI 1971. Mais la déclinaison des Cinq Noms nous amène naturellement à élargir la perspective. Ils sont en fait au nombre de cinq ou six : أَب 《père », أَ 《frère », حَ 《beau-père »,

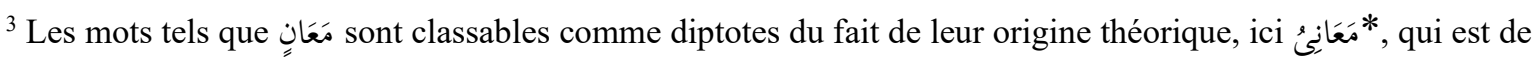
forme diptote normale. La déclinaison donnée l'est par la plupart des grammaires, dont celles de WRIGHT 1985, FISCHER 1987 et SILVESTRE de SACY 1831a; mais certaines mentionnent cependant un génitif indéterminé en -iya. BROCKELMANN 1909, p. $25^{*}-28^{*}$, a omis de traiter ces mots dans ses tables.
} 
ذُو 《possesseur de», فُ ف le sixième de ces mots est هَ "chose », qui peut aussi suivre la déclinaison triptote normale. Les Cinq Noms constituent une déclinaison triptote spéciale : aux formes de l'état indéterminé, pourvues de nunation, s'opposent bien des formes sans nunation qui constituent globalement l'état déterminé ; mais elles sont de deux types :

- d'une part des formes terminées par une voyelle longue qui apparaissent (en première approximation à nouveau) si et seulement si le mot a un complément en annexion. Nous pouvons dire que ces formes à voyelle longue des Cinq Noms constituent leur état construit pour autant que nous donnions à l'état construit, et donc à l'état inconstruit, une portée générale en les redéfinissant ainsi :

l'état construit (resp. inconstruit) est la réunion des situations où les pluriels masculins externes et les duels perdent (resp. conservent) leur u final ;

- d'autre part des formes à voyelle brève finale sans nunation qui apparaissent lorsque les Cinq Noms sont à l'état déterminé, mais sans complément en annexion. Ces formes sont donc celles d'un état à la fois déterminé et inconstruit.

La déclinaison des Cinq Noms est ainsi intermédiaire entre celle des mots qui fonctionnent sur l'opposition indéterminé / déterminé, et celle de ceux qui le font sur l'opposition inconstruit / construit ${ }^{4}$.

Dans la mesure où nous avons retenu (provisoirement) comme seule situation d'état construit la présence d'un complément en annexion au mot considéré, et du fait que cette situation figure parmi les situations d'état déterminé, nous pouvons dire que l'état construit implique l'état déterminé ; et qu'inversement, l'état indéterminé implique l'état inconstruit. Par conséquent, les seules combinaisons possibles des états construit, inconstruit, déterminé et indéterminé sont ces trois états, exclusifs les uns des autres : indéterminé, construit, et déterminé inconstruit ; l'état indéterminé construit est impossible.

On peut donc présenter la déclinaison des Cinq Nom, soit en mentionnant les couples d'états déterminé/indéterminé et construit/inconstruit, soit en indiquant uniquement leurs combinaisons :

\begin{tabular}{|c|c|c|c|}
\hline & Indéterminé & \multicolumn{2}{|c|}{ Déterminé } \\
\hline $\mathrm{N}$ & أَبُْ & أَبُ & أَبَُو \\
\hline G & أَبِ & أَبِ & أَبِي \\
\hline A & أَبًَا & أَبَب & أَبَا \\
\hline & \multicolumn{2}{|c|}{ Inconstruit } & Construit \\
\hline
\end{tabular}

\footnotetext{
${ }^{4}$ Selon Wright 1985, vol. I, p. 250 A, et Silvestre de SACY 1831a, p. 417, l'état construit de بِنْت 《fille » peut être إِنْة (d'après l'ajout inséré dans Wright, cette dernière forme était même préférée anciennement en toute situation, sauf en début de phrase). Il s'agit donc d'un mot fonctionnant potentiellement lui aussi sur base de ces trois états.
} 


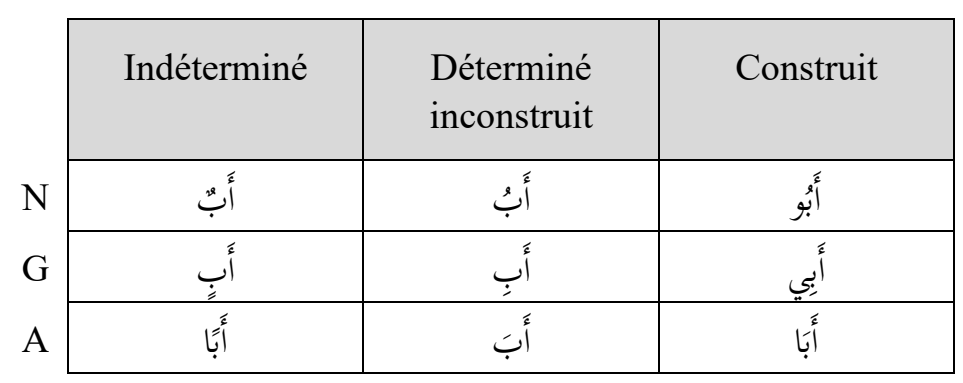

Ces présentations peuvent être étendue aux autres déclinaisons; ainsi, comme représenté ci-dessous, celle des diptotes normaux, avec les trois états exclusifs les uns des autres. Cette perspective à trois états est dopté pour l'arabe (avec d'autres dénominations) dans l'excellente grammaire de FISCHER 1987, parue pour la première fois en 1972, ainsi que dans la réflexion théorique de RETSÖ 1984-1986 et 2010.

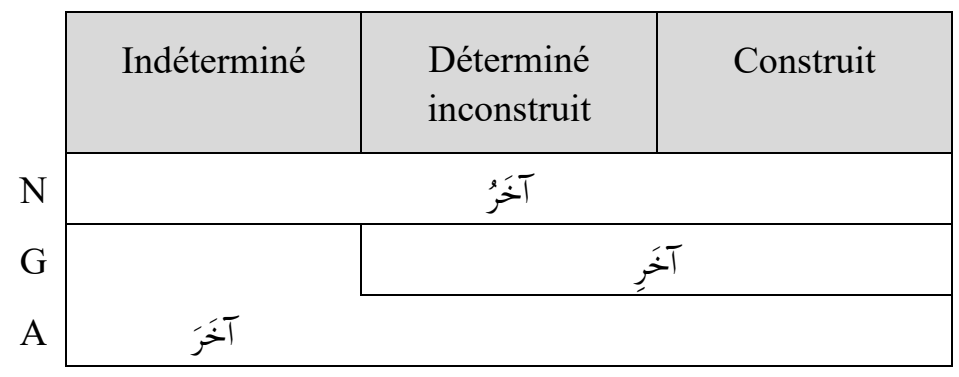

Or il est à noter que l'état construit est celui du mot qui a un complément en annexion (en fait, pas uniquement, comme nous le verrons dans la seconde partie) ; que l'état indéterminé est celui du mot qui n'a ni article ni complément en annexion (pourvu qu'il ne soit introduit ni par le لَ de négation absolue ni par ou de ses analogues); et que l'état déterminé inconstruit est notamment celui du mot qui porte l'article. On retrouve donc en arabe, quoique de façon un peu plus sophistiquée, la structure en trois états exclusifs les uns des autres qu'on retrouve notamment en araméen : l'état construit (status constructus); l'état du nom ou adjectif sans article, qui correspond à notre état indéterminé (appelé status indeterminatus, status absolutus), et l'état où le mot est pourvu de l'article - $\hat{a}$, que celui-ci ait ou non une réelle fonction de définition ${ }^{5}$. Comme cet article ne peut pas s'adjoindre à un mot en état construit, ce troisième état correspond à notre état déterminé inconstruit ; pour ne pas nous démarquer des appellations utilisées à propos de l'araméen, il semble donc indiqué de l'appeler état emphatique, s'il faut lui donner un nom particulier.

\section{Propriétés remarquables des déclinaisons. Bon ordre des cas et des états}

Considérons d'abord les tableaux à deux états, déterminé/indéterminé et construit/inconstruit. Quelle que soit la langue concernée, l'ordre adopté pour les cas essaie normalement de regrouper les cas qui donnent lieu à des formes identiques dans l'une ou l'autre déclinaison. Pour ce qui est de l'arabe, c'est l'ordre nominatif-génitif-accusatif (qui se trouve rejoindre celui habituellement adopté pour les déclinaisons du grec ancien) qui permet de regrouper les formes identiques dans chaque déclinaison. Ceci est rendu possible par le fait qu'il n'existe pas en arabe de déclinaison où, pour un état donné, les formes du nominatif et

\footnotetext{
${ }^{5}$ En araméen occidental et en vieil araméen, le mot en l'état nommé généralement état déterminé ou emphatique est de sens défini.
} 
de l'accusatif soient identiques entre elles mais différentes de celle du génitif. Dans chaque état, les plages occupées sur nos tableaux par chaque forme distincte de chaque déclinaison sont donc d'un seul tenant.

En outre, une seconde propriété se dégage : dans chaque déclinaison, quand on fusionne les plages identiques éventuelles des états concernés par cette déclinaison, comme nous l'avons fait, les plages obtenues sont rectangulaires, à l'exception remarquable de la plage de la forme de type tionnée dans notre note 3 , de la déclinaison diptote en -in). De celle-cí, on peut seulement dire qu'elle est d'un seul tenant. La raison de cette propriété est que, dans les déclinaisons où des formes identiques apparaissent dans les états concernés (qui sont les états déterminé et indéterminé, puisque ces déclinaisons sont celles des triptotes défectueux et des diptotes), les formes se regroupent de la même manière dans les deux états - sauf pour ces diptotes.

Quant aux tableaux à trois états exclusifs les uns des autres, comme on le voit, si l'on adopte pour les états l'ordre indéterminé-emphatique-construit, l'état déterminé se trouve constitué par les colonnes contiguës de l'état emphatique et de l'état construit; de même, l'état inconstruit est constitué des colonnes contiguës de l'état indéterminé et de l'état emphatique ${ }^{6}$. Par conséquent, avec pour les cas l'ordre nominatif-génitif-accusatif décrit plus haut, les plages occupées par chaque forme distincte de chaque déclinaison dans la perspective à trois états restent d'un seul tenant. Avec ce bon ordre des cas et des états, nous pouvons étendre aux tableaux à trois colonnes la constatation faite à propos de ceux à deux colonnes : les plages sont toutes des rectangles (et même des rectangles sans trou, précision qui n'a d'intérêt que pour la plage unique des indéclinables, qui occupe les neuf cases du tableau), sauf celles de type آخَرَ de de la variante de la déclinaison des diptotes en $-i n^{7}$. Nous approfondirons l'étude de ces propriétés dans la seconde partie.

\section{Tableau synoptique des déclinaisons}

On peut également établir un tableau synoptique montrant la sensibilité de chaque déclinaison aux états, comme ci-dessous (tout comme on pourrait le faire de leur sensibilité aux cas) ${ }^{8}$. Les indéclinables ont évidemment été laissés de côté, de même, pour la raison évoquée

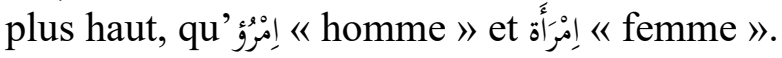

\footnotetext{
${ }^{6}$ L'intérêt de cette façon de disposer les états échappe à RETSÖ 1984-1986, p. 343, et 2010, p. 70 ; il adopte l'ordre indéterminé-construit-emphatique, aussi bien qu'à Fischer (op. cit., pp. 80-81).

${ }^{7}$ Les diverses propriétés que nous reconnaissons aux tableaux correspondent à des propriétés générales de structure bien connues en topologie mathématique : convexité (réduite ici au fait d'être rectangulaire et sans trou), connexité (fait d'être d'un seul tenant), connexité simple (fait d'être d'un seul tenant et sans trou).

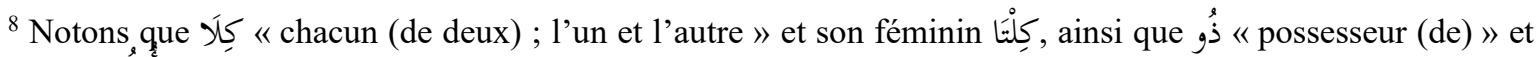

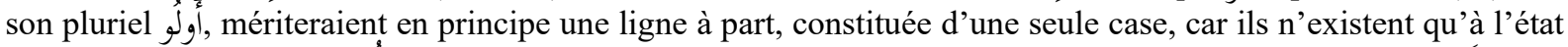

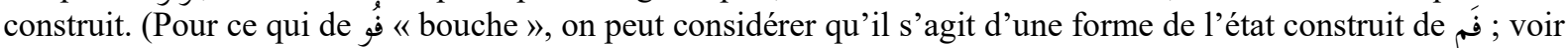
WriGHT 1985, vol. I, p. 249 B).
} 


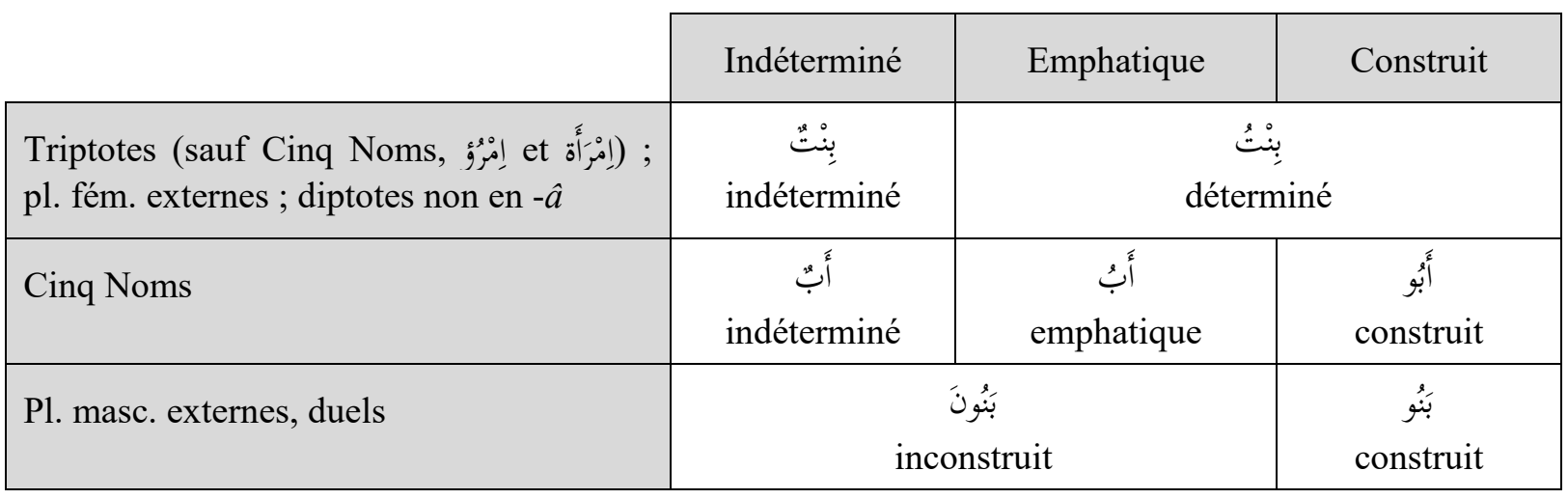

\section{Détermination / définitude. Simplification du vocabulaire}

En préambule notamment au passage en revue des diverses appellations données aux cas, il y a un point qu'il nous faut préciser. Nous avons évoqué en introduction l'intérêt d'une clarification du vocabulaire. Dans divers ouvrages traitant de grammaire arabe, il est question de deux déterminations : la détermination grammaticale ou par la forme, et la détermination sémantique ou par le sens ${ }^{9}$. La seconde, la détermination sémantique, est ce qu'on nomme de nos jours définitude : le fait d'être défini, c'est-à-dire de sens défini. (On parle aussi de définitude positive pour un mot de sens défini, et de définitude négative - ou d'indéfinitude - pour un mot de sens indéfini). Nous adoptons ce vocabulaire, ce qui nous laisse face à une seule détermination : la détermination grammaticale, qui donc sera simplement pour nous la détermination ${ }^{10}$. Et ce qui est en détermination (grammaticale) sera dit déterminé. Cette acception du mot « déterminé » coïncide en fait avec celle que nous en avions proposée plus haut à propos des états, car la détermination grammaticale n'est autre que ce que nous avons appelé « état déterminé ».

Cette perspective nous paraît mieux adaptée à la description de la langue arabe que le langage habituel, car elle permet de condenser en un mot des formules diverses et lourdes : " déterminé quant au sens », " déterminé grammaticalement», "de sens défini », " de sens déterminé », etc. Cette lourdeur a trop souvent pour conséquence que, pour alléger l'expression, on se contente d'utiliser les mots « déterminé » et « défini » dans un sens vague, sans préciser s'il s'agit de détermination sémantique ou de détermination grammaticale. Cette ambiguïté aboutit à une véritable confusion dans certaines publications.

Et cela jusque dans la grammaire de WRIGHT 1985. Defined et undefined y correspondent généralement à " déterminé » et « indéterminé » (grammaticalement). Mais, parfois, defined doit être compris comme «(rendu) défini »; et undefined, comme «indéfini », « non rendu défini », voire « vague ». Les termes determined, determination et apparentés sont employés de manière encore moins précise. Par contre, cet ouvrage utilise nettement definite et indefi-

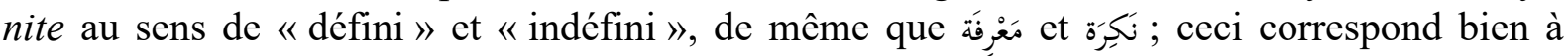

\footnotetext{
${ }^{9}$ BROCKELMANN 1941, notamment, utilise le mot « determiniert » à la fois pour la détermination grammaticale, ainsi dans les tableaux de déclinaisons p. $25^{*}-28^{*}$, et pour la détermination sémantique (« an und für sich determiniert », p. 68-69.

${ }^{10}$ Les mots tels que « détermination », « déterminé », « déterminant » s'utilisent généralement de nos jours à propos de faits de construction de phrase, indépendamment du sens. Il y a cependant plusieurs perspectives. JACQUESSON 2016, p. 6, utilise «détermination » au sens de : fait d'avoir un complément [en annexion], une épithète, un démonstratif, ainsi qu'il l'écrit p. 6, mais ne considère pas l'article comme un déterminant (c.à.d. élément de détermination). SARTORI 2019, à propos de l'arabe, donne à « détermination » un sens encore différent. Et MARTIN 1966, p. 11, donne à ce mot un sens tout autre ; voir WILMET 1985, pp. 219-220.
} 
l'acception habituelle de ces mots arabes selon les dictionnaires et d'autres grammaires ${ }^{11}$. Cependant, en un passage (vol. II, p. 260 D), indefinite et نكرِة sont exceptionnellement à comprendre comme « indéterminé », ainsi que l'explique une note. Ceci montre peut-être que la distinction entre détermination (grammaticale) et définitude n'était déjà pas aussi nette qu'on le croit chez les grammairiens arabes traditionnels ${ }^{12}$.

La définitude est une notion suffisamment importante pour que nous insérions ici quelques précisions à son sujet. Grosso modo, en arabe comme en français et en d'autres langues, est défini ce qui est présenté comme connu du lecteur ou auditeur, ou du rédacteur, notamment les noms pourvus de l'article défini (seul article existant en arabe) et, habituellement, les noms propres. Mais bien qu'elle soit un élément sémantique, la définitude joue un rôle syntaxique en arabe, distinct de celui de la détermination; d'où la nécessité de ne pas les confondre ${ }^{13}$. La définitude y est liée notamment, comme en hébreu, à une règle d'accord en définitude entre l'épithète et le nom auquel celle-ci se rapporte, étant entendu qu'un adjectif est

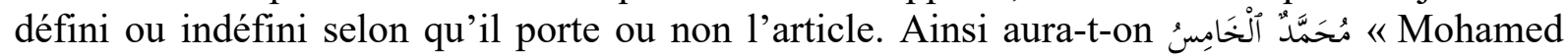

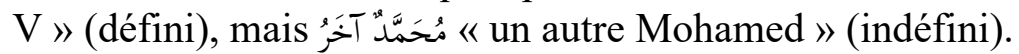

Notons que si le vocabulaire des linguistes maintient l'usage de ces mots, détermination et définitude, que l'on tendrait à confondre, c'est parce que l'on reconnaît et rappelle ainsi qu'il existe effectivement une relation entre eux : dans certaines situations, ces deux idées vont de pair. Mais ce n'est pas toujours le cas ; d'où, aux yeux de certains, concernant l'arabe, une impression d'incohérence dont nous allons reparler à propos de la terminologie. En fait, comme le montrent les exemples simples repris dans le tableau suivant (où nous prenons la détermination au sens particulier que nous lui avons donné), toutes les combinaisons de détermination et de définitude existent en arabe. De même le fait qu'un mot arabe soit en état construit ou inconstruit ne permet-il pas à lui seul de savoir si ce mot est défini ou indéfini.

\begin{tabular}{|c|c|c|}
\hline & Indéfini & Défini \\
\hline Indéterminé & 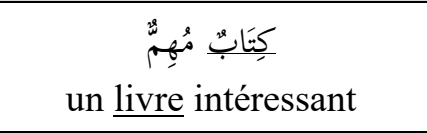 & $\begin{array}{l}\text { مُحَمََّّدُ أْخَخَامِسن } \\
\text { Mohammed V }\end{array}$ \\
\hline Déterminé & 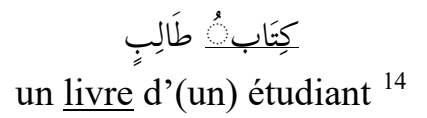 & $\begin{array}{c}\text { كِتَابُ ألطَّالِبِ } \\
\text { le livre de l'étudiant }\end{array}$ \\
\hline
\end{tabular}

Cette indépendance vis-à-vis de la définitude n'existe pas pour tous les états dans toutes les langues sémitiques : en araméen occidental et en vieil araméen, l'utilisation de l'état « emphatique » ou " déterminé » implique la définitude ; de même, évidemment, celle de l'état pronominal d'un nom, quelle que soit la langue sémitique (en particulier en akkadien, hébreu,

${ }^{11}$ Notamment Al-Nûr ..., p. 26, et, dans l'Encyclopedia of Arabic Language and Linguistics, les articles Nunation (vol. III, pp. 453-454) de F.-C. MUTH et Tanwîn (vol. IV, pp. 442-446) de G. AYOUB.

12 Notons aussi qu'en Wright, vol. I, p. 247 C, un insert entre crochets, non dû à Wright, traduit فَعْرَ et نكِرَة par defined et undefined, mots anglais qui viennent d'être présentés par Wright lui-même, p. 247 B, d'une manière qui correspond à « déterminé » et « indéterminé ». Mais l'auteur de l'insert était peut-être moins précis que WRIGHT dans sa manière de s'exprimer.

${ }^{13}$ La définitude et l'indéfinitude constituent donc elle-mêmes des éléments de certaines situations grammaticales.

${ }^{14}$ Le style habituel du français répugne à utiliser une expression telle qu' « un livre d'un étudiant » et la remplace par «le livre d'un étudiant» ou par « un livre d'étudiant». 
guèze, dans lesquels cet état se distingue de l'état construit ordinaire ${ }^{15}$ ), puisque le complément en annexion est alors défini par nature, ce qui entraîne la définitude du nom sur lequel il porte ${ }^{16}$.

Notons encore que ce débat de terminologie concerne en fait un problème de fond qui se retrouve ailleurs que dans les études arabes, quels que soient les termes utilisés : la distinction à maintenir clairement entre la définitude et l'indéfinitude, d'une part, et de l'autre les états (dont l'état emphatique), situations grammaticales qui impliquent certaines formes des noms et adjectifs. Il ne semble malheureusement pas si rare de rencontrer, ou d'avoir à soupçonner, une confusion entre «état déterminé » (c.à.d. état emphatique) et définitude, ou entre " état indéterminé » et indéfinitude ${ }^{17}$.

\section{Cas / état}

Un fait qui ressort de cette présentation est une complémentarité entre cas et état. Le travail que nous avons effectué pour cerner la notion d'état peut se faire pour les cas, et aboutit de même à une définition à deux niveaux : désignant au départ les formes des triptotes normaux classées selon le timbre de leur dernière voyelle (nominatif pour les formes où la finale est de timbre $u$, c'est-à-dire $-u$ ou -un, etc.), les définitions des cas, pour pouvoir être appliquées à d'autres mots que les triptotes normaux, doivent être étendues aux réunions de situations grammaticales où apparaissent les formes triptotes normales liées à ces cas ${ }^{18}$. Cas et états sont donc, finalement, des réunions de situations grammaticales. Mais alors que le cas d'un mot est lié à sa fonction, c'est-à-dire à la manière dont il se rapporte à d'autres mots de la phrase (normalement à un seul autre mot, en fait), son état l'est en gros à la manière dont d'autres mots de la phrase se rapportent à lui : article, complément du nom ou de l'adjectif, complément d'objet direct lorsqu'il s'agit d'un participe actif (détail que nous étudierons dans la seconde partie), compléments et autres termes « limitatifs » lorsqu'il s'agit d'un mot introduit par et ses analogues ${ }^{19}$.

Autrement dit, dans une vue hiérarchisée où tout part de la base de la phrase (verbe pour une phrase verbale, mubtada' pour une phrase nominale), le cas dépend de ce qui est en amont du mot; et l'état, de ce qui est en aval. Voir ci-dessous l'exemple de la phrase

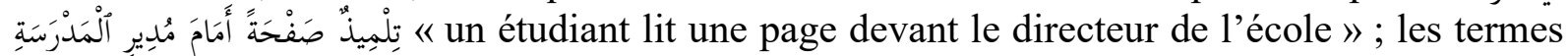
apparaissent de droite à gauche dans l'ordre chronologique de leur énonciation, tandis que les flèches vont de chaque mot à ce qui se rapporte à lui. Si par exemple nous considérons le mot مُدِير, son cas est fixé par sa relation au mot qui le précède, أَمَّام, et son état l'est par le fait que

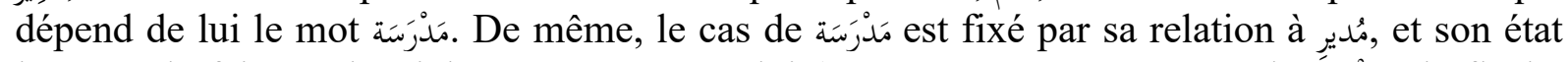

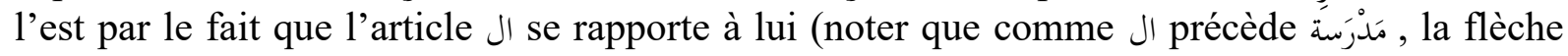
entre ces deux mots va de gauche à droite, en sens inverse du temps).

${ }^{15}$ Voir RETSÖ 2010, p. 65. En fait, dans ces langues, l'état construit se subdivise en un état construit nominal (dit simplement état construit), où le mot en annexion est un nom (ou un adjectif, en arabe en tout cas), et un état construit pronominal (dit simplement état pronominal), où ce mot est un pronom affixe.

${ }^{16}$ Voir HAELEWYCK 2016, p. 163, [709].

17 Ainsi à propos de l'hébreu biblique, VAN DER MERWE 1999, p. 4 et 187, qui désigne par status determinatus la définitude (positive). Notons que VyCICHL 1969 (ibid.) envisage pour l'arabe plusieurs types de status determinatus et de status indeterminatus liés à la fois à la forme et au sens.

${ }^{18}$ Cf. l'article Case Theory de Mark S. LeTourneau, p. 347, dans l'Encyclopedia of Arabic Language and Linguistics.

${ }^{19}$ Concernant يَ et ses analogues, voir Wright, vol. II, p. 85 et ss. Mais la situation, là, est complexe car la définitude semble y entrer aussi en jeu, ainsi que l'intention du locuteur. 


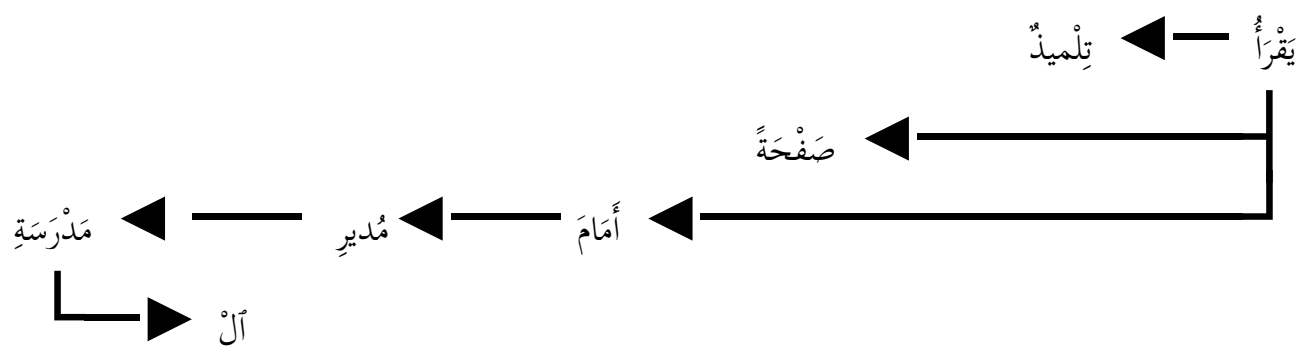

Certains éléments étrangers à l'environnement des mots jouent parfois un rôle dans la situation grammaticale concernée : c'est le cas dans la syntaxe de et de ses analogues, où la

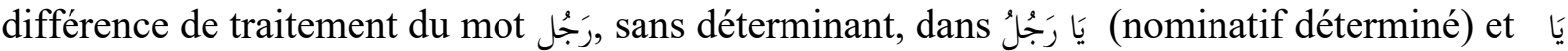
رَجِلًَ (accusatif indéterminé) " ô homme » dépend de ce que l'on s'adresse ou non à une personne précise et censée pourvoir percevoir réellement l'appel.

Cette façon de voir les choses semble valable également pour les autres langues sémitiques : l'état y est bien lié à ce qui se rapporte, ou ne se rapporte pas, au nom ou adjectif concerné ; le cas, pour les langues qui connaissent les déclinaisons, est lié à ce à quoi ce mot se rapporte. (Il y a cependant au moins une exception : le status praedicativus qui se rencontre occasionnellement en akkadien).

\section{Utilité des états dans la formulation de règles grammaticales}

Certaines règles peuvent se formuler de façon plus concise grâce aux états. Ainsi peut-on écrire que :

- le mot sur lequel porte le $\bar{y}$ de négation absolue utilisé de la façon habituelle ${ }^{20}$ est à l'accusatif déterminé. Cet accusatif sera soit déterminé inconstruit (c'est-à-dire empha-

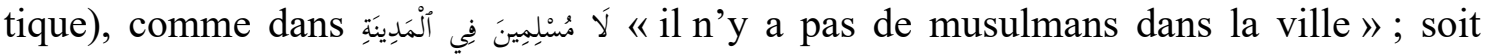

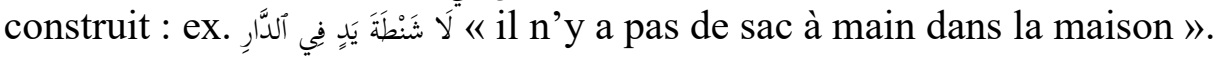

- s'il désigne une ou plusieurs personnes précises, réelles et censées pouvoir percevoir l'appel et que rien ne se rapporte à lui, le mot introduit par (ou un de ses analogues ${ }^{21}$ ) se met au nominatif emphatique, c'est-à-dire déterminé, mais inconstruit - ce qui est

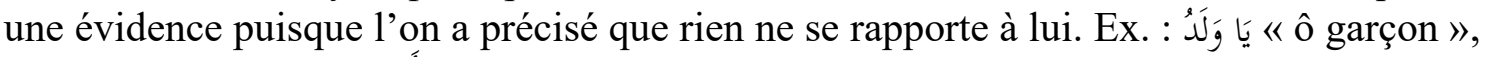

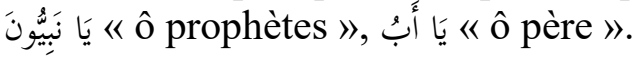

Ces formulations condensées sont particulièrement intéressantes dans les listes de vocabulaire.

Introduisons ici une remarque à propose du لَ de négation absolue et de ses analogues. On voit que ces particules conditionnent non seulement le cas du mot qu'elles introduisent, ce qui est normal, mais aussi leur état. La raison pourrait en être qu'elles jouent un rôle analogue à celui d'un article, et qu'on peut donc les voir comme se rapportant au mot qu'elles introdui-

${ }^{20}$ Nous résumons ici par « façon habituelle » les situations où le mot introduit par le لَ de négation absolue est à l'accusatif (pour les exceptions, facultatives ou obligatoires, voir WRIGHT, vol. II, pp. 95 B et 97 B-98 A) et où rien ne se rapporte à lui, épithètes et compléments d'objet direct logiques introduits par ل mis à part (voir WRIGHT, vol. II, pp. 96 C-97 B).

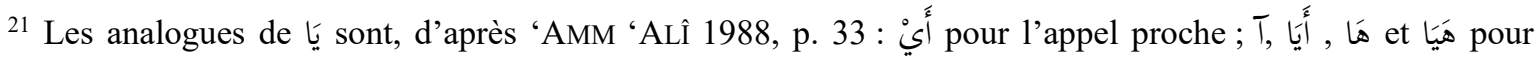
l'appel lointain; tandis que sert aussi bien à l'appel proche qu'à l'appel lointain. WRIGHT, vol. II, p. 85 A, ne cite que أيًا et, mais ces particules semblent au complet dans WRIGHT, vol. I, pp. 294 B et 295 D (où il est question d'une règle particulière pour 1 gَ et). 
sent alors que lui-même se rapporte à elles. Au niveau de l'arbre de la phrase, ceci se traduirait par l'apparition une boucle ; ainsi dans لَا شَخْصَ فِي ألدَّارِ il n’y a personne dans la maison ».

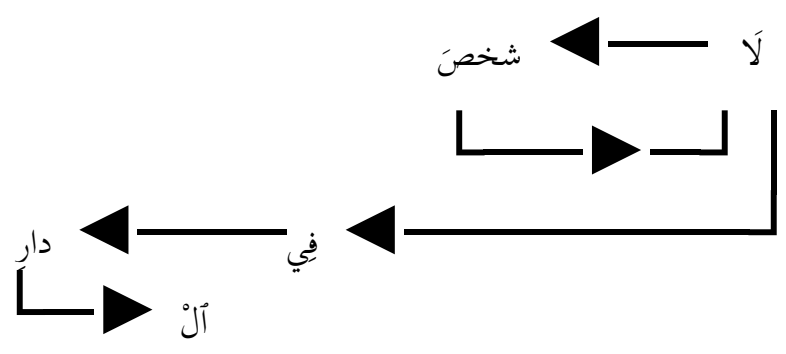

\section{Terminologie}

Comme on le voit aux précautions de langage que nous avons prises, il y a un problème de terminologie. Les divers états repris ci-dessus apparaissent bien dans la littérature, mais sous des appellations diverses ; celles utilisées par les uns se retrouvent parfois avec un sens différent chez les autres.

La perspective des premiers grammairiens de l'hébreu à avoir utilisé la notion d'état était bien celle que nous avons décrite. L'état absolu (status absolutus, qui correspond à notre état inconstruit) et l'état construit, celui-ci sous le nom de status regiminis, apparaissent avec REUCHLIN 1506 ; BUXTORF 1629 semble le premier à signaler qu'on nomme aussi ce dernier status constructus ${ }^{22}$. Concernant l'arabe, WRIGHT utilise l'expression status constructus dans le même sens que nous, et précise (vol. II, p. 198) que tel est l'usage des grammairiens européens. Concernant l'hébreu, c'est aussi ce qui ressort de VAN HECKE (voir n. 1). Un survol de l'Internet à propos de l'emploi de cette expression en grammaire de l'arabe et de l'hébreu paraît confirmer que les expressions état construit, construct state, status constructus sont le plus souvent utilisées de cette façon.

Mais en fait, comme l'a relevé RETSÖ, la notion d'état est très peu présente dans les ouvrages concernant la grammaire de l'arabe, et souvent limitée à la mention de l'état construit ; en outre, une tradition parallèle s'est établie qui, pour l'arabe et rarement pour l'hébreu, nomme « état construit » la relation ou structure d'annexion elle-même ${ }^{23}$. Tel est notamment le cas, pour l'arabe, de BLACHÈRE 1978, p. 322, et de l'Encyclopedia of Arabic Language and Linguistics (VERSTEEGH 2005-2008) ${ }^{24}$. En particulier, dans cette dernière, l'article 'Iḍ̂ffa, p. 294, considère que l'expression construct state est normalement utilisée en anglais pour rendre le mot 'ị̂afa des descriptions traditionnelles de la grammaire arabe, concurremment

\footnotetext{
${ }^{22}$ Voir n. 1. Nous lisons en effet chez REUCHLIN 1506, p. 558 : «Ut autem quid dicam de regimine et affixis intelligas, consyderare debes utriusque numeri duplicem statum. unus est absolutus, alter status regiminis », et chez Buxtorf (1629), pp. 325-3266, premier grammairien connu à avoir utilisé le terme de status constructus : «Forma haec vocatur vulgò Status constructus, Status regiminis, vel simpliciter Regimen : Formam autem integram Nominis extra constructionem positi, vocant Statum absolutum. »

${ }^{23}$ Voir RETSÖ 2010, p. 66. Notons aussi que RECKENDORF 1921, p. 135, mentionne l'état construit, mais le comprend comme étant le premier terme de l'annexion, non l'état dans lequel ce mot se trouve.

${ }^{24}$ L'expression construct state ne semble apparaître dans l'Encyclopedia of Arabic Language and Linguistics qu'aux articles Construct state de Benmamoun, p. 477-482, et 'Ilâfa de RYDing et VersteEgh, p. 294-298. Notons que JACQUESSON 2016 utilise « état construit » tantôt au sens que nous étudions (p. 4, 5), tantôt au sens de la relation ou construction d'annexion au sens habituel (p. 3, 4, 5, 6), et étend même le terme à certaines constructions avec préposition, mais de même sens (p. 5).
} 
avec d'autres : genitive construct, construct phrase, annexation structure. La notion d'état que nous étudions semble par contre totalement ignorée de cette encyclopédie ${ }^{25}$.

L'état construit, en grammaire de l'arabe, est généralement connu sous ce nom (status constructus, construct state, etc.) Certains le nomment pourtant autrement : état radical (status radicalis) pour VYCICHL 1969, p. 211 ; état II pour RETSÖ 1984-1986, p. 343, et 2010, p. 70. Notons aussi que quelques auteurs utilisent l'expression «état construit » pour le premier terme de certaines autres constructions de sens similaire, à deux termes également ${ }^{26}$. Quant à l'état inconstruit, à l'exception de BISHAI 1971, p. 48, qui l'appelle état absolu (absolute state) comme en hébreu biblique, il ne semble pas porter en général de nom particulier en grammaire de l'arabe.

L'état indéterminé est souvent appelé par ce nom (status indeterminatus). BISHAI, p. 23, l'appelle état non restreint (non-restricted state). D'aucuns (mais non BISHAI : voir ci-dessus) le nomment aussi état absolu (status absolutus), en grammaire de l'arabe et d'autres langues. Ceci, vraisemblablement, pour ce qui est de l'arabe, parce que les noms communs et adjectifs pris indépendamment de tout contexte, dans l'absolu, sont généralement cités au nominatif indéterminé, notamment dans les entrées de la plupart des dictionnaires actuels : بَبنُنَ, بِنْنْ etc.

Notre état déterminé, quant à lui, correspond au status determinatus de TSERETELI 1991, p. 1571-1572, qui le définit comme le contraire de l'état indéterminé dans le cadre de l'araméen biblique; se démarquant ainsi de la définition habituelle du status determinatus, ainsi que nous allons le voir. BISHAI (ibid.) l'appelle état restreint (restricted state).

Nous avons nommé état emphatique l'état déterminé inconstruit pour les raisons déjà exposées. L'autre appellation fréquente de cet état, utilisée notamment par FISCHER pour l'arabe, est status determinatus, qu'il semblerait naturel de traduire par «état déterminé », mais ce serait inapproprié. En effet, status determinatus et status indeterminatus sont ici à comprendre comme état défini et état indéfini ${ }^{27}$. La raison en est que, comme nous l'avons vu plus haut, les noms communs et adjectifs en status indeterminatus sont en principe indéfinis, tandis qu'ils sont définis quand ils portent l'article (en arabe, mais aussi en hébreu, en araméen occidental, en vieil araméen) ${ }^{28}$, ce qui est la principale des situations reprises dans le status determinatus de FISCHER, c'est-à-dire l'état emphatique.

Un défaut évident de ce vocabulaire est que l'on attendrait que ces états défini et indéfini soient contraires l'un de l'autre, alors que ce n'est pas le cas : le nom en état construit n'est ni en état défini, ni en état indéfini. On donne donc l'impression de fonctionner sur une logique à trois termes : vrai, faux, incertain. En outre, on peut se trouver en présence de mots qui sont à la fois de sens défini et en état indéfini, مُحَمَّمَ par exemple, ou de sens indéfini et en état défini,

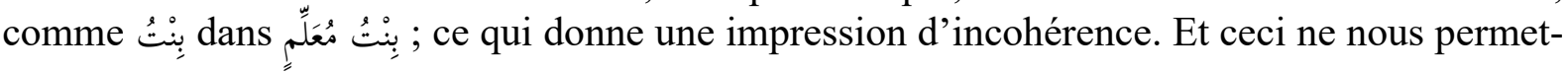

\footnotetext{
${ }^{25}$ Si ce n'est que l'article 'Id̂afa, p. 296, note : « Some grammarians also consider a noun followed by a possessive pronoun suffix to be in the construct state (see, for instance Fischer 2002 : 89) ». Ses auteurs pensent ici à l'état pronominal, qui peut se distinguer de l'état construit dans certaines langues (voir plus haut, n. 14).

${ }^{26}$ LECOMTE 1976, p. 84, considère comme en état construit le démonstratif suivi du nom qui lui est apposé. JOMIER 1983, p. 8-9 et 37, parlent d'état construit ou d'état d'annexion en grammaire du dialecte du Caire pour tout nom suivi d'un complément déterminatif direct ou introduit par betấ'

${ }^{27}$ L'état qui n'est ni construit ni déterminé est de fait souvent appelé état défini en français ; par exemple par JACQUESSON, op. cit., p. 4, à propos de l'araméen ancien, pour lequel cette appellation est justifiée car le nom en cet état y est nécessairement de sens défini.

${ }^{28}$ On peut (à l'instar de JACQUESSON, ibid., à propos de l'araméen occidental et du vieil araméen) considérer que la finale du mot $(-\hat{a},-\hat{o})$ est l'article; et en conséquence, que c'est sur la forme du mot privé de l'article que repose l'état en question.
} 
trait plus de simplifier le vocabulaire comme proposé plus haut, puisque dire simplement d'un mot qu'il est défini, ou indéfini, deviendrait ambigu. Pour ces raisons, nous choisissons de parler d'état emphatique plutôt que d'état défini. L'arabe n'est évidemment pas la seule langue concernée par ces remarques.

Cette impression d'incohérence, concernant l'arabe, mérite que nous nous y attardions un instant. Elle provient essentiellement d'une perception erronée de la nunation comme sorte d'article indéfini, en pendant de l'article défini آ Cette perception fait voir comme anormale, notamment, l'existence de noms propres triptotes de sens défini mais portant la nunation,

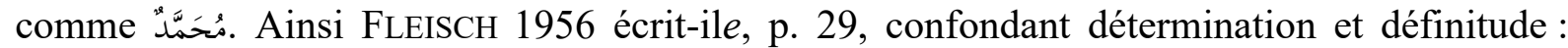
« comment admettre qu'un nom propre, déterminé autant qu'un nom puisse l'être, reçoive un suffixe d'indétermination? " Ceci peut mener loin, jusqu'à suggérer, comme l'ont fait FERRANDO 2000, p. 40-41, et TROUPEAU 2008, que les déclinaisons arabes sont une pure invention de grammairiens. Il convient évidemment de renverser la perspective : ce que montrent ces prétendues anomalies, c'est que la nunation n'est pas un article indéfini en arabe. On ne peut affirmer le contraire que si l'on ferme les yeux sur certains faits, le traitement des noms propres sans article notamment. La question de sa signification précise et de son origine peut certes être posée, mais ne remet pas en question la structure de la langue arabe telle qu'elle nous est parvenue ${ }^{29}$.

Pour terminer ce passage en revue - qui ne se prétend pas exhaustif -, récapitulons ici sous forme de tableau les principales dénominations que nous avons rencontrées à propos des états ; les qualificatifs latins sont en italiques.

\begin{tabular}{|l|c|c|c|c|c|}
\cline { 2 - 6 } \multicolumn{1}{c|}{} & Tsereteli & Fischer & Bishai & Retsö & autres \\
\hline Indéterminé & indeterminatus & $\begin{array}{c}\text { indeterminatus } \\
(=\text { indéfini })\end{array}$ & non-restreint & I & absolutus \\
\hline Déterminé & determinatus & restreint & absolu & II & radicalis \\
\hline Inconstruit & constructus & constructus & construit & III & emphaticus \\
\hline Construit & & $\begin{array}{c}\text { determinatus } \\
(=\text { défini })\end{array}$ & & & \\
\hline Emphatique & & & & & \\
\hline
\end{tabular}

\section{Conclusion de la première partie}

Pour la pratique courante, sur le plan pédagogique, on peut se contenter de l'exposé qui précède. Il en ressort que les déclinaisons arabes ont une structure commune qui leur permet d'être présentées dans des tableaux clairs facilitant leur apprentissage. Les appellations que nous proposons pour les divers états sont celles qui nous paraissent les plus adaptées, tenant le juste milieu entre la tradition orientaliste et la terminologie linguistique actuelle. Mais quelle que soit la perspective et le vocabulaire adoptés, la notion d'état, pour autant qu'elle soit utilisée de manière cohérente - notamment en ce qui concerne la nunation et la définitude -, per-

\footnotetext{
${ }^{29}$ RETSÖ 2010, p. 67-68, étudie longuement la controverse sur le rôle de la nunation. Lequel semble en fait avoir été, à l'origine, de marquer l'état inconstruit, à en croire LIPIŃSKI 1997, p. 272.
} 
met de mieux saisir l'organisation des déclinaisons arabes. Elle fournit aussi un vocabulaire simple et précis pour l'analyse des problèmes grammaticaux, permettant notamment une formulation condensée.

Cette étude a produit aussi quelques résultats théoriques. Elle a montré la symétrie profonde entre les notions de cas et d'état; l'une comme l'autre désignent, à la base, des formes de noms et adjectifs, et doivent être étendues à des réunions de situations grammaticales qui entraînent ces formes. Elle a également établi l'existence pour l'arabe d'un ordre naturel des cas, qui fait apparaître deux propriétés de structure de toutes les déclinaisons arabes à l'exception des diptotes normaux (et de la variante des diptotes en -in). Elle a établi de même, concernant la perspective à trois états, l'existence d'un ordre naturel de ceux-ci, qui permet de maintenir la manifestation des propriétés structurelles dans les tableaux à trois cas et trois états. Nous poursuivrons dans la seconde partie cette étude en revenant sur certaines questions apparemment marginales mais a priori susceptibles de mettre en péril ce bel édifice.

\section{Perspective approfondie}

Dans notre première partie, nous avons laissé en suspens quelques points qui ne sont pas indispensables pour cerner la nature et l'utilité des états. Nous en traitons maintenant:

- la redéfinition que nous avons donnée des états déterminé et indéterminé fonctionne en général pour la description des déclinaisons diptotes. Mais il y a quand même une situation qui pose problème : la forme des mots suivis de بْن dans l'énoncé d'une filiation.

- nous avons assimilé l'état construit à la présence d'un complément en annexion. Mais il y a deux autres situations, peu connues il est vrai, où apparaissent aussi les formes de l'état construit.

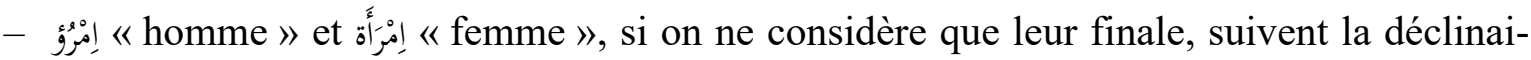
son triptote normale. Mais la forme de ces deux mots change généralement quand on les

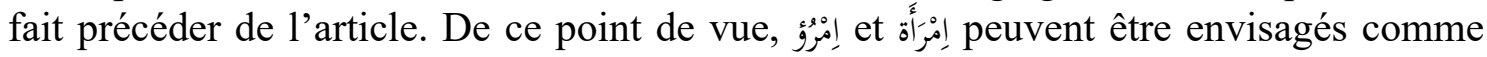
constituant une déclinaison particulière.

Le système des états peut-il intégrer ces trois points en gardant sa cohérence ? Telle sera notre question. En outre, la prise en compte des deux situations additionnelles d'état construit appellera une remarque sur la prononciation de l'état construit des mots terminés par a.

\section{Le nom propre suivi de بْن dans une filiation}

La grammaire arabe, celle de WRIGHT en tout cas, considère implicitement que l'état par défaut des triptotes et des féminins externes (ainsi que du nominatif et du génitif des diptotes en -in) est celui où ils sont pourvus de la nunation : l'état indéterminé. Ce sont donc les situations d'état déterminé, de perte de nunation, qui font l'objet de règles et que nous devons rechercher si nous voulons vérifier que notre définition généralisée des états déterminé et indéterminé s'applique bien partout. Cette recherche peut être complétée par celle de propos concernant la déclinaison diptote, que WRIGHT appelle aussi second declension.

L'examen de ces situations révèle que dans l'une d'elles, notre généralisation de l'état déterminé sur base des triptotes ne rend pas compte du comportement des diptotes. La situation qui fait problème est celle-ci :

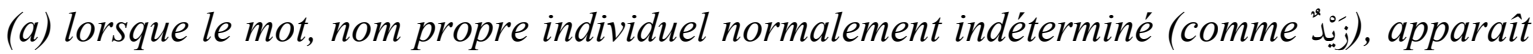
comme nom de fils dans une succession de deux noms propres indiquant une généalogie de père en fils dans laquelle il est suivi immédiatement de إِبْن " fils 》 (qui prend alors la forme 
sauf en début de ligne ou si le père est désigné par un surnom ${ }^{30}$ ) : ainsi زَيَّد بْنُ عَمْرِ (Zayd fils de 'Amr».

Cette situation est assez singulière : il ne s'agit pas seulement de construction de phrase ; la signification de l'expression et le fait qu'il s'agisse ou non d'un nom propre interviennent également. La règle, telle qu'énoncée par WRIGHT (vol. I, p. $249 \mathrm{~B}$ et $250 \mathrm{~A}$ ), est encore plus singulière que la situation elle-même : les triptotes perdent leur nunation éventuelle; ainsi - بْنُ عَمْرِو

Remarquons d'abord que l'on trouve une règle différente chez BlACHÈRE 1975, p. 124. Pour eux, le nom propre dans la situation $(a)$ est à l'état construit. Ceci aurait notamment pour conséquence qu'au génitif, les diptotes normaux dans la situation (a) prendraient un - $i$ final, non un - $a$, puisqu'ils seraient dans la forme de l'état que nous avons appelé état déterminé. Ni eux ni WRIGHT ne donnent d'exemple permettant de vérifier cela, mais le Hadith en fournit en

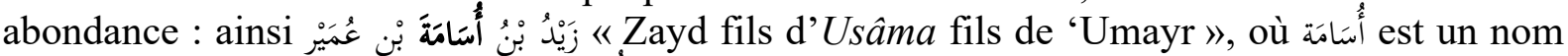
d'homme diptote ${ }^{31}$. On voit que أُمَّامَ a bien ici la finale - $a$ du génitif indéterminé, conformément à la formulation de WRIGHT. La règle donnée par BLACHÈRE 1975 n'est donc pas valide.

Par ailleurs, l'énoncé de WRIGHT n'est pas aussi clair qu'on pourrait le penser. Celui-ci considère en fait comme relevant de la déclinaison triptote toutes les formes avec nunation (voir vol. I, p. 246 D-247 A). La chute de la nunation doit donc se produire non seulement pour les triptotes, mais aussi pour les éventuels noms propres individuels qui, comme envisagé par SîBAWAYHI, suivraient la déclinaison des diptotes en $-i^{32}$ ou des pluriels féminins externes ${ }^{33}$. La règle semble plutôt pouvoir s'exprimer ainsi : dans la situation (a), les formes censées porter la nunation perdent celle-ci.

Cette nouvelle formulation pose cependant encore un problème à propos des triptotes et diptotes défectueux. Dans la situation (a), les noms propres individuels triptotes en -an doivent donc voir leur finale passer de $\varsigma^{\prime}$ à $\mathfrak{s}^{\prime}$; les choses, ici, semblent claires ${ }^{34}$. Mais qu'en estil des triptotes et diptotes en -in ? Je ne dispose pas d'exemple concret concernant leur existence, si ce n'est que Sîbawayhi traite de l'utilisation éventuelle de (forme de « huit 》servant à dénombrer des féminins) comme nom propre d'homme diptote ${ }^{35}$. Si l'on comprend notre dernier énoncé de la règle de Wright au sens de «les formes censées avoir la nunation voient celle-ci remplacée par le signe de voyelle brève correspondant », ثَمَانِ passe à dans la situation (a); cette forme ne correspondrait à aucun couple (cas, état) des déclinaisons en in. Mais à voir ce que veut dire Wright lorsqu'il écrit, en d'autres circonstances, qu'un triptote en -in ou diptote en -in perd sa nunation (comme en vol. I, p. 247 D), on attendrait plutôt

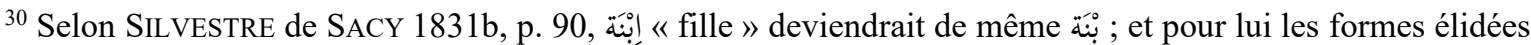
et بْنَ بْنَ vaudraient quand ces mots connectent deux noms propres, sans plus de précision.

${ }^{31}$ English Translation (...), p. 33.

${ }^{32}$ Sîbawayhi, p. 19, 1. 22 (Jahn, p. 272).

${ }^{33}$ Pour les noms propres d'hommes et de femmes relevant de la déclinaison des féminins pluriels externes, voir SÎBAWAYHI 1970, p. I ^, 11. 8-12 (JAHN 1900, p. 269-270). Un ajout à WRIGHT, vol. I, p. 243 B, signale effectivement des pluriels féminins externes utilisés comme noms propres individuels et conservant leur déclinaison, mais semble ne concerner que des noms individuels féminins. Pour les diptotes en -in, voir SîBAWAYHI, p. 1 9, 1. 22 (JAHN, p. 272).

${ }^{34} \mathrm{Il}$ n'en existe pas en principe (voir WRIGHT, vol. I, p. 242 C D), mais les diptotes en -â sont censés passer à la déclinaison triptote en -an lorsqu'ils sont pris dans un sens générique (WRIGHT, vol. I, p. 245 A). Je ne dispose malheureusement pas d'exemple de ce type.

35 Noter qu'étonnamment, SîBAWAYHI (ibid.) explique la diptosie du nom propre s'agirait d'un mot féminin dans l'usage ordinaire (triptote), alors que ce mot est fondamentalement masculin (WRIGHT, vol. I, p. 255 A).
} 
c'est-à-dire l'état déterminé. Dans cette perspective, la règle devrait s'énoncer : les formes censées avoir la nunation passent à l'état déterminé. Nous manquons d'exemples permettant de trancher. Mais, du point de vue de l'oral - c'est-à-dire, en fait, de la langue -, nos deux derniers énoncés sont équivalents. La forme déterminée ثَنَّمَانِي comme ثَمَانِ, du fait de la liaison avec $b n^{36}$. Nous adopterons la seconde de ces deux formulations, parce qu'elle nous permet de laisser de côté la question de l'orthographe. Elle nous permet en outre de nous exprimer en termes d'états.

La perspective de WRIGHT, selon laquelle les mots dans la situation (a) sont naturellement à l'état indéterminé, sauf certains qui y sont à l'état déterminé, peut être renversée à partir de la définition de principe de l'état déterminé, qui est basée sur le comportement des triptotes normaux. Puisque ceux-ci sont à l'état déterminé dans la situation $(a)$, il est logique de considérer que tous les mots dans cette situation sont naturellement à l'état déterminé, sauf certains, qui y sont à l'état indéterminé. De ce point de vue, la seule déclinaison à faire exception est la déclinaison diptote normale, dont nous avons discuté plus haut. Nous pouvons donc proposer comme règle que, dans la situation (a), les mots sont à l'état déterminé, à l'exception des diptotes normaux, qui sont à l'état indéterminé. Ou encore, en cernant au plus près le problème, que les diptotes normaux censés être au génitif ont la finale -a, et qu'en dehors de cela, les mots sont à l'état déterminé. Ceci toujours, bien entendu, avec la remarque faite plus haut sur l'orthographe des éventuels triptotes ou diptotes en -in.

On le voit, cette dernière formulation est prudente concernant l'interprétation de la finale $a$ des diptotes normaux. Il y a en effet deux manières d'essayer de décrire celle-ci en termes de cas et d'état :

- soit considérer que le mot est bien à l'état déterminé, mais à l'accusatif ;

- soit considérer qu'il est bien au génitif, mais à l'état indéterminé.

On fait donc dépendre de la déclinaison tantôt le cas, tantôt l'état. Aucune de ces deux solutions n'est satisfaisante : dans la première, c'est la définition des cas qui est mise à mal ; dans la seconde, celle des états. L'une et l'autre se basent en effet, en principe, sur le comportement des triptotes. (Accessoirement, ceci souligne une fois encore la complémentarité entre cas et état).

Notons que les mots insensibles à l'opposition déterminé / indéterminé sont diversement assignés à l'un ou l'autre de ces deux états selon la règle adoptée, et ceci bien sûr sans que leur forme en soit affectée. Il s'agit des noms propres individuels diptotes normaux au nominatif et à l'accusatif, diptotes en -in à l'accusatif, indéclinables et, comme envisagé par Sîbawayhi, pluriels masculins externes et duels ${ }^{37}$.

En conclusion, remarquons que, si l'on fait abstraction de la variante des diptotes en -in, c'est à nouveau la déclinaison diptote normale qui fait cavalier seul, ainsi que nous l'avions déjà remarqué à propos de la structure des tableaux de déclinaisons.

\section{Les trois situations d'état construit}

La forme normale des pluriels masculins externes et des duels, chez WRIGHT, est implicitement celle de l'état inconstruit. C'est donc l'état construit qui y fait l'objet de règles que nous pouvons rechercher. On découvre ainsi qu'il en est question dans trois situations :

\footnotetext{
${ }^{36}$ Voir WRIGHT, vol. I, p. 21 B.

${ }^{37}$ p. I ᄉ, 11. 8-12 (JAHN, p. 269-270).
} 
(b) lorsque le mot a un complément en annexion - c'est la situation signalée plus haut, à laquelle on croit souvent que se réduit l'état construit - ; et cela même si, dans l'usage moderne notamment, ce complément en annexion n'est pas exprimé parce que réapparaissant

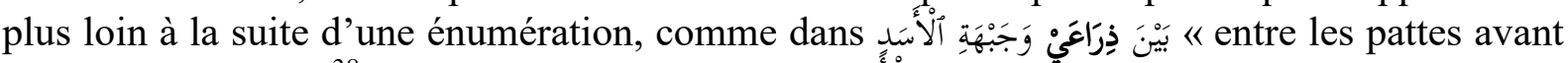

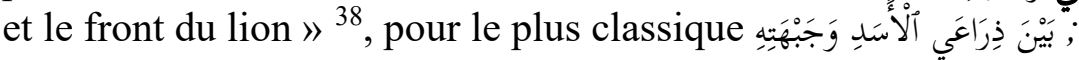

(c) lorsque le mot est introduit par le لَ de négation absolue utilisé de la façon habituelle (cf. supra) et précède (pas forcément immédiatement) la préposition ل d'appartenance,

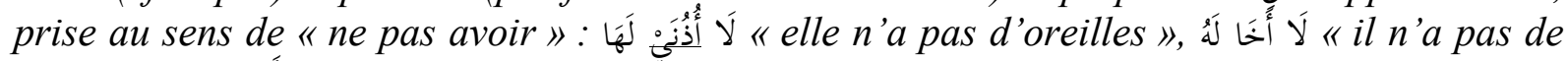
frère » (mais أَب " père » peut aussi être à l'état inconstruit, donc emphatique, dans cette si-

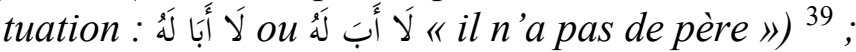

(d) facultativement, lorsque le mot est un participe actif à sens inaccompli, est muni de

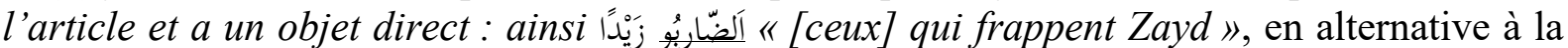
formulation courante الَضَّاربُونَ زيَّْدَ et à celle où l'objet direct devient complément en annexion formelle : 40.

Pour bien comprendre les différentes constructions qui sont possibles dans cette situtation (d), qui n'a évidemment d'intérêt que si le participe est au masculin pluriel externe ou au duel, il convient de se rappeler que les participes actifs à sens inaccompli ont à la fois valeur de verbe et d'adjectif. En tant que verbes, ils peuvent avoir un complément d'objet direct à l'accusatif : الكَّاربُونَ زيَّْدا. Mais, comme adjectifs, ils peuvent aussi transformer celui-ci en com-

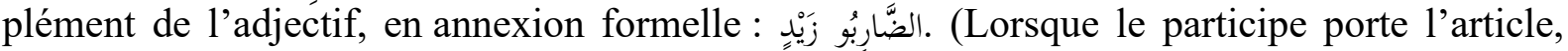
cette transformation n'est possible au singulier que si ce complément en annexion est défini

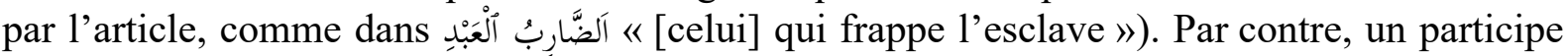
actif à sens accompli est simplement un nom; de ce fait, son complément d'objet direct logique ne peut apparaître que sous la forme d'un complément déterminatif, en annexion réelle,

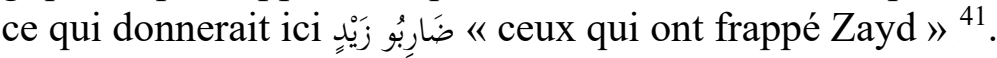

Remarquons que l'état construit apparaît aussi dans la partie des unités du cardinal 12 (إثنًا (إثْنَّا عَشْرَةَ ,عَشَرَ $)$, laquelle est un duel. Ce cas particulier ressortit vraisemblablement à la situation (b), le complément devant être عَشْرَةَ ou Celui-ci est en annexion formelle puisque l'expression est de sens défini ou indéfini selon qu'elle commence ou non par l'article : الثْنَا عَشَرَ

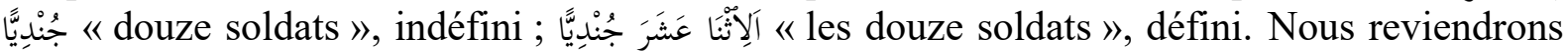
plus loin sur cette question.

Examinons maintenant les deux nouvelles situations. La première, (c), entraîne les formes de l'état construit: WRIGHT le dit explicitement et fournit à l'appui de cette règle des exemples tirés de toutes les catégories dont la forme dépend de ce que l'on soit ou non en état construit : pluriels masculins externes, duels et Cinq Noms ${ }^{42}$. La seconde de ces nouvelles

${ }^{38}$ WRIGHT, vol. II, p. 201 B C. DiCHY 2000, p. 107 n., signale que cette construction est maintenant intégrée par les manuels et l'enseignement.

${ }^{39}$ Voir Wright, vol. II, p. 95 C D.

${ }^{40}$ Wright, vol. II, pp. 66 A-67 A.

${ }^{41}$ Pour ce paragraphe, voir WRIGHT, vol. II, pp. 64 B-65 A, outre les pp. 66 A-67 A déjà mentionnées. On notera que les participes à sens inaccompli, ayant donc valeur d'adjectifs, forment en principe un pluriel externe ; voir BORRMANS 1967, p. 25 et 28b, et Wright, vol. I, pp. 195 A , 195 C D et 197 C.

${ }^{42} \mathrm{La}$ formulation de WRIGHT, vol. II, p. $95 \mathrm{C}$ D, ne dit pas clairement si l'état construit est ici obligatoire ou facultatif. Concernant les Cinq Noms, la manière dont il expose le cas de أَبْ montre bien qu'il s'agit pour lui d'une obligation au moins pour أَ أَبَ est, seule autre instance citée des Cinq Noms : « la forment, aussi utilisée devant لِ (notre trad.) Comme Wright écrit plus généralement que le nom qui précède لِ est en état construit dans cette situation, il semble bien qu'il s'agisse d'une règle et qu'elle doive valoir pour tous les noms 
situations, $(d)$, est exprimée par Wright pour les pluriels masculins externes et les duels, mais la troisième catégorie concernée par l'état construit, les Cinq Noms, ne contient aucun participe : on peut donc décrire cette situation comme nous l'avons fait, sans exclure a priori les Cinq Noms.

Par ailleurs, les situations (b), (c) et (d) sont des situations d'état déterminé puisque les triptotes et les pluriels féminins externes y perdent leur nunation. Nous le savons déjà pour

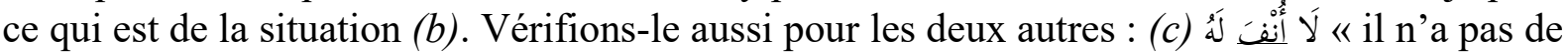

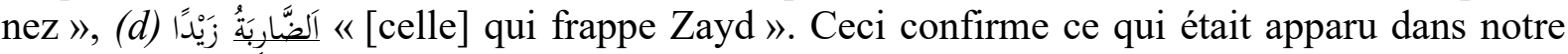
première partie, quand nous ne prenions pas en compte les situations $(c)$ et $(d)$ : même en incluant ces nouvelles situations, l'état construit reste un sous-état de l'état déterminé ; inversement, l'état indéterminé reste un sous-état de l'état inconstruit. Les combinaisons possibles des états restent donc bien les états indéterminé, emphatique et construit; et le tableau synoptique montrant la sensibilité des déclinaisons aux états n'est pas perturbé.

Et tout cela, soulignons-le, du fait que la situation (d) contient un détail qui ne semble guère prévisible : que le participe doive y porter l'article. Il semblerait pourtant a priori possible d'imaginer qu' «ils frappent Zayd» puisse se dire هُمْ ضَارِبُو زَيْدًا. Avec pour complément d'objet direct ce type de nom propre en tout cas, il n'y aurait pas d'ambiguïté sur le fait que

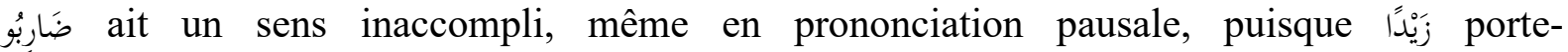
rait indéniablement la marque de l'accusatif (prononcée - $\hat{a}$ à la pause) - chose impossible s'il avait un sens accompli, comme vu plus haut. La raison de la nécessité de l'article ne paraît donc pas à chercher dans la signification de l'expression. Le lecteur intéressé trouvera dans notre annexe 1 ce que deviendrait le tableau synoptique à trois états en l'absence de cette propriété providentielle.

Par ailleurs, nous avions vu dans la première partie que les états permettaient de formuler certaines règles de façon concise. Quel est l'impact sur ces formulations de la prise en compte des nouvelles situations $(c)$ et $(d)$ ?

- pour le لَ de négation absolue utilisé de la façon habituelle, la règle en elle-même n'est pas modifiée : le mot sur lequel porte ce لَ est à l'accusatif déterminé. Cependant, l'état

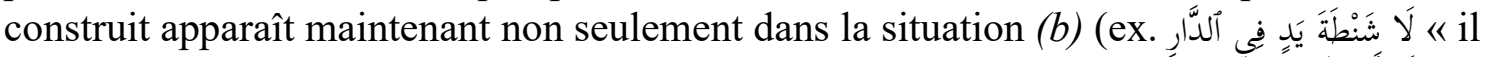

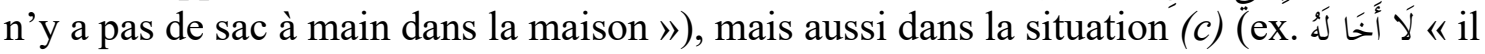
n'a pas de frère »). Quant à la situation $(d)$, elle n'est pas envisageable puisque le parti-

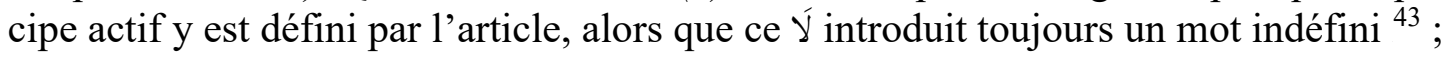

- la règle concernant يَ et ses analogues n'est évidemment pas concernée par la situation (c). Elle ne l'est pas non plus par la situation $(d)$, puisque le mot doit à la fois y porter l'article et avoir un complément d'objet direct, alors que cette règle suppose que rien ne se rapporte au mot concerné.

concernés par l'état construit. On peut d'ailleurs remarquer que parmi les exemples donnés par WRIGHT se

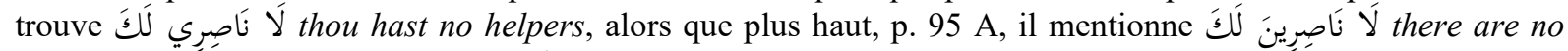
helpers for thee. Dans le second cas, ل J'exprime pas l'appartenance mais l'avantage ou le c.o.d. logique : c'est la différence de sens qui explique la différence de construction. Ceci paraît donc bien confirmer que l'état construit est de règle dans la situation (c).

${ }^{43}$ Wright, vol. II, p. 98 C D. 


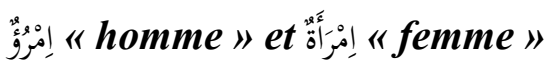

Venons-en à ces deux triptotes qui se présentent habituellement sous une forme qui dépend de ce qu'ils ont ou non l'article ${ }^{44}$. On peut très bien les décrire sans invoquer la notion d'état : la finale de ces mots suit la déclinaison des triptotes normaux. Mais on peut aussi con-

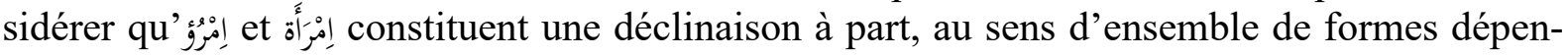
dant des cas et des états. Il est intéressant de constater qu'ils s'intègrent alors parfaitement dans notre panorama. Dire qu'un mot porte ou non l'article, c'est dire qu'il est en état avec article ou en état sans article; nous avons bien affaire ici à des états, avec la dualité que nous avons rencontrée jusqu'ici : forme prise par le mot, mais aussi manière dont ce mot est précisé par ce qui se rapporte à lui - en l'occurrence l'article éventuel.

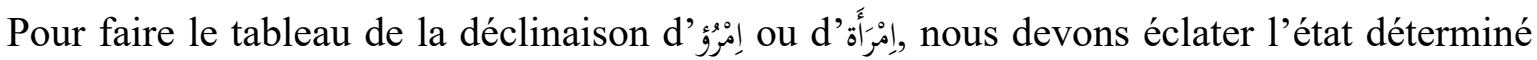
des triptotes entre état déterminé avec article (c'est-à-dire état avec article, simplement) et état déterminé sans article ; l'état indéterminé est évidemment sans article. Comme avec les Cinq Noms, nous nous retrouvons donc avec trois états : état indéterminé, état avec article, et état déterminé sans article. Voici deux façons de présenter la déclinaison de إِمرُو (qui se trouve, lorsqu'il est sans article, être aussi à déclinaison double, c'est-à-dire que sa voyelle interne varie de la même façon que sa voyelle de déclinaison ${ }^{45}$ ) ou celle de إِمرَأَ :

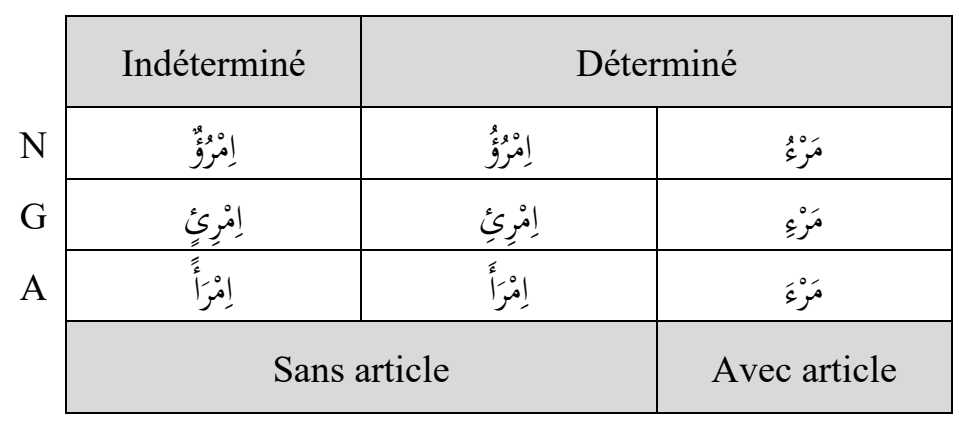

\begin{tabular}{|c|c|c|c|}
\hline & Indéterminé & $\begin{array}{l}\text { Déterminé } \\
\text { sans article }\end{array}$ & Avec article \\
\hline $\mathrm{N}$ & إِمْرَ|ََة & إِمرَأَأَُ & مَرَأَةًُ \\
\hline G & إِمرَأَةٍ & إِمَرَأَة & مَرْأَة \\
\hline A & إِمَرَأًََ & إِمَرَأَةَّة & مَرْأَةً \\
\hline
\end{tabular}

44 A lire BLACHÈRE 1975, p. 30, on pourrait croire qu'il en est de même de أنَّاس "ن gens 》; mais ce n'est pas le cas. Ce mot, dans lequel certains voient l'origine de son synonyme نَّاس peut très bien s'utiliser avec

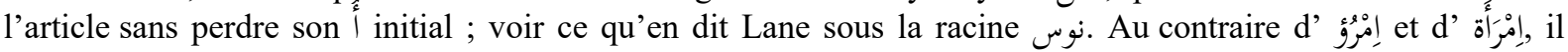
commence en outre par un hamza stable.

45 J'ai opté ici pour l'orthographe logique de ces mots, ainsi que pour la déclinaison non double en ce qui concerne مَزْ

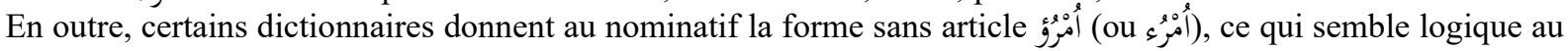
vu de la règle de la voyelle du hamza initial dans les impératifs ; mais ce n'est pas ce qui est rapporté par Lane. 
L'état déterminé sans article peut se rencontrer, pour ces deux mots, à l'état construit

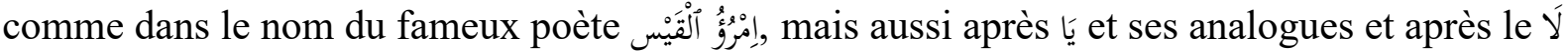
de négation absolue ${ }^{46}$.

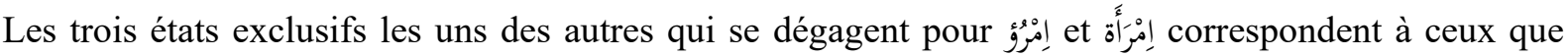
l'on trouve pour les autres noms et adjectifs arabes en ce sens que, reprenant le tableau précédent au

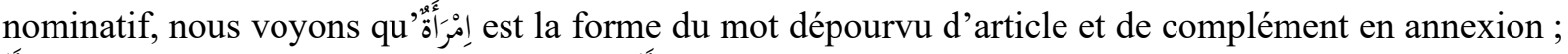

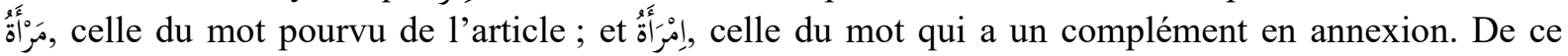
point de vue, on pourrait donc considérer l'état avec article comme un état emphatique spécial et l'état

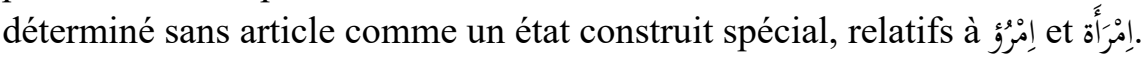

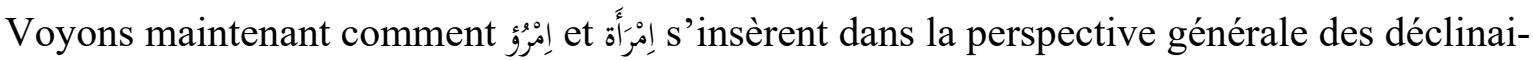
sons. On peut reprendre la question au départ de la perspective à trois états, indéterminé / emphatique / construit. Comme vu plus haut, l'état indéterminé reste tel quel : il est nécessaire-

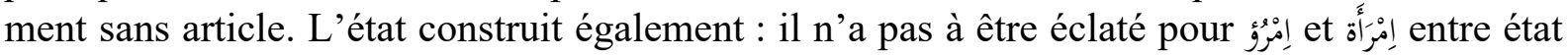
construit avec article et état construit sans article, car il s'agit de noms. Au contraire des adjectifs, en effet, les noms ne peuvent en même temps porter l'article et être à l'état construit ${ }^{47}$. A l'état construit, إمْرَأَّ pont donc nécessairement sans article.

Par contre l'état emphatique peut se distinguer, pour ces deux mots, entre état emphatique

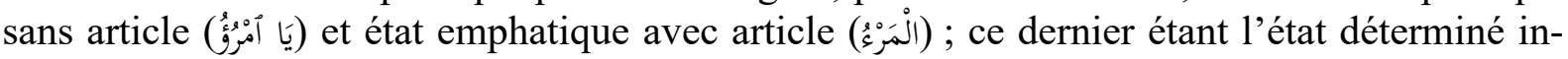
construit avec article, donc simplement l'état inconstruit avec article. En fait, pour les noms

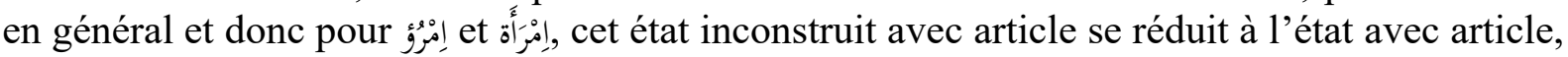
puisque - comme nous venons de le rappeler - un nom qui porte l'article ne peut avoir de complément en annexion. C'est pourquoi le mot « inconstruit » apparaît entre crochets dans le tableau qui suit (écrit pour le nominatif seulement); nous l'y maintenons pour pouvoir aussi appliquer plus tard cette grille de lecture aux adjectifs. Noter que le fait de placer l'état emphatique sans article à droite de l'état inconstruit avec article nous permet d'assurer l'unité de

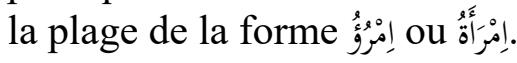

Au-dessous du tableau, nous avons repris les états tels qu'ils apparaissaient dans les tableaux précédents.

\begin{tabular}{|c|c|c|c|}
\hline Indéterminé & $\begin{array}{l}\text { [Inconstruit] } \\
\text { avec article }\end{array}$ & $\begin{array}{l}\text { Emphatique } \\
\text { sans article }\end{array}$ & Construit \\
\hline 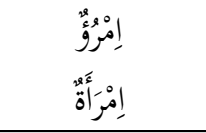 & مَرَّرُة & \multicolumn{2}{|c|}{ 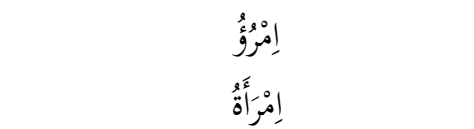 } \\
\hline Indéterminé & Avec article & \multicolumn{2}{|c|}{ Déterminé sans article } \\
\hline
\end{tabular}

Il se dégage ainsi une perspective à quatre états élémentaires qui peut être utilisée pour la présentation de n'importe quelle déclinaison, en ce compris cette fois celles d' إمْرَأَ propriétés des déclinaisons vues dans notre première partie s'y manifestent nécessairement aussi. La déclinaison des Cinq Noms, par exemple, peut être présentée ainsi :

\footnotetext{
${ }^{46}$ Ceci en principe. Mais dans les récitations du poème لا امرأة إلا أنت de Nizâr QABBÂNI trouvées sur You-

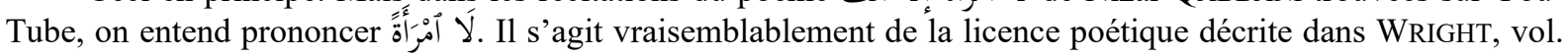
II, p. 388 A.

${ }^{47}$ Cf. Wright, vol. II, pp. 198 C - 199 A.
} 


\begin{tabular}{|c|c|c|c|}
\hline Indéterminé & $\begin{array}{l}\text { [Inconstruit] } \\
\text { avec article }\end{array}$ & $\begin{array}{l}\text { Emphatique } \\
\text { sans article }\end{array}$ & Construit \\
\hline أَبُْ & \multicolumn{2}{|c|}{ أَبُ } & أَبَّو \\
\hline أَبِ & \multicolumn{2}{|c|}{ أَبِ } & أَبَي \\
\hline أَبًَا & \multicolumn{2}{|c|}{ أَبَ } & أَبًا \\
\hline
\end{tabular}

Ces quatre états nous permettent à nouveau d'établir un tableau synoptique de la sensibilité des déclinaisons aux états, comme nous l'avions fait plus haut sans avoir encore envisagé et إمرَزَأَ Voici un tel tableau, en laissant bien sûr à nouveau de côté les indéclinables :

\begin{tabular}{|c|c|c|c|c|}
\hline & Indéterminé & $\begin{array}{l}\text { Inconstruit } \\
\text { avec article }\end{array}$ & $\begin{array}{l}\text { Emphatique } \\
\text { sans article }\end{array}$ & Construit \\
\hline 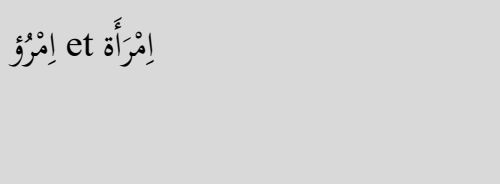 & $\begin{array}{c}\text { Iإمْرُوٌْ } \\
\text { indéterminé }\end{array}$ & $\begin{array}{c}c_{80}^{8} \\
\text { avec article } \\
\text { (emphatique spécial) }\end{array}$ & \multicolumn{2}{|c|}{$\begin{array}{c}\text { إِمْرُؤُ } \\
\text { déterminé sans article } \\
\text { (construit spécial) }\end{array}$} \\
\hline 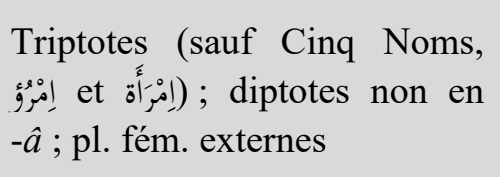 & indéterminé & \multicolumn{3}{|c|}{ 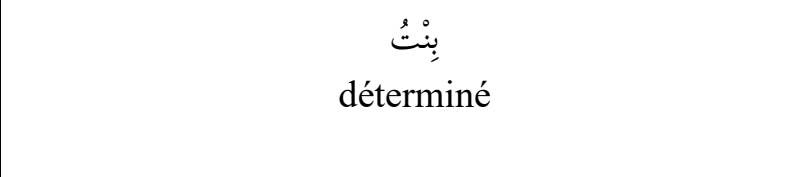 } \\
\hline Cinq Noms & $\begin{array}{l}\text { أَبٌْ indéterminé } \\
\text { indé }\end{array}$ & \multicolumn{2}{|c|}{ emphatique } & $\begin{array}{c}\text { أَبُو } \\
\text { construit }\end{array}$ \\
\hline Pl. masc. externes, duels & \multicolumn{3}{|c|}{$\begin{array}{c}\text { inconstruit } \\
\text { inنُونَ }\end{array}$} & $\begin{array}{c}\text { بَنُو construit } \\
\text { بَو }\end{array}$ \\
\hline
\end{tabular}

Comme dans le tableau synoptique que nous avions obtenu précédemment, décrivant les déclinaisons autres que celles de ces deux mots, chaque état caractéristique de chaque groupe de déclinaison occupe une plage d'un seul tenant (et même rectangulaire puisqu'il s'agit ici de combinaisons de rectangles sur une même ligne du tableau); et il n'y a aucune case vide, de sorte que le tableau synoptique lui-même est un rectangle. La cohérence que nous avions observée dans les déclinaisons est donc maintenue même lorsque إِمْرُو et ent pris en considération.

Soulignons que ces belles propriétés ne vont pas de soi : elles seraient mises à mal si إِمْرٔ et إمْرَأَة

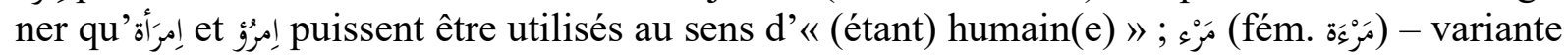

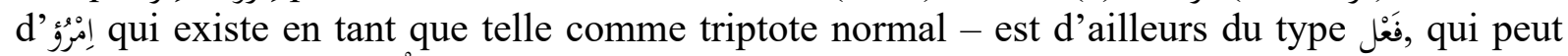

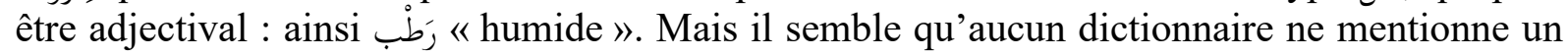
tel sens.

Quoi qu'il en soit, ce tableau nous donne l'occasion de faire quelques réflexions globales sur les déclinaisons arabes : 
- il y a quatre types de fonctionnement des mots par rapport aux états, ou cinq si l'on prend en compte les indéclinables, pour quatre états élémentaires (dont un seul n'apparaît qu'en combinaison avec d'autres états : l'état emphatique sans article);

- à chaque cas, les noms et adjectifs ont trois formes, éventuellement confondues : celle de l'état indéterminé, celle de l'état inconstruit avec article, et celle de l'état construit ;

- les indéclinables mis à part, aucune déclinaison ne fonctionne sur un seul état ;

- les indéclinables mis à part, il y a une seule déclinaison qui fonctionne sur un seul cas, en ce sens que nominatif, génitif et accusatif y sont confondus : celle des triptotes en an, tel مَعْنَ « signification ».

\section{État construit et prononciation}

Ajoutons encore que l'opposition état inconstruit / état construit semble intervenir aussi, actuellement du moins, dans la prononciation des mots terminés par un $a$ bref suivi de a lorsque, dans une prononciation simplifiée, on laisse tomber la finale de déclinaison de ces mots. Lorsqu'un tel mot est suivi d'un complément en annexion, le a est alors généralement prononcé $t^{48}$. Ainsi entendra-t-on مَدْرَسَة جَمِيلَة madrasa jamîla 《 une belle école », mais مَدْرَسَة الْفْنُون madrasat alfunûn « l'école des arts », par exemple.

Notons d'ailleurs que dans la partie des unités des numéraux cardinaux et ordinaux de 11 à 19 servant à dénombrer des masculins, le áventuel de cette partie subsiste, prononcé $t$, dans les formes dialectales de ces nombres ; 15 devient ainsi hamstâš en Algérie. Ceci suggère que cette partie des nombres de 11 à 19 est à l'état construit, à l'instar de ce qui s'observe dans le

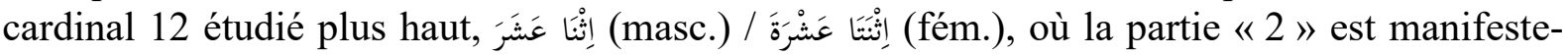
ment à l'état construit et se décline (alors que la partie des unités semble figée à l'accusatif dans les autres nombres de cette catégorie) ${ }^{49}$.

Tout ceci pourrait s'exprimer en termes d'états : le aterminant un mot en état construit se prononce t. Cela pour autant, en principe, que cette prononciation se manifeste également dans les situations $(c)$ et $(d)$ vues plus haut, c'est-à-dire que les prononciations courantes de,

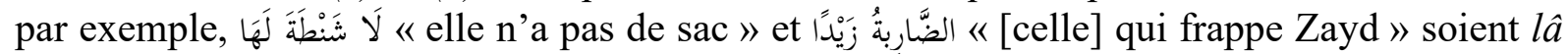
$\check{s}$ antat lahâ et, facultativement, add âribat zaydan. Comme cette condition est vraisemblablement devenue impossible à vérifier de nos jours, nous pouvons en pratique la considérer comme validée, et donc présenter les choses comme suit :

48 TAlloen 2007, p. 46 et 83 ; WightwiCK 1990, p. 32 ; HAELEWYCK 2016, p. 153. Noter qu'il se passe presque la même chose en hébreu, non seulement dans la prononciation des mots féminins, mais dans l'écriture :

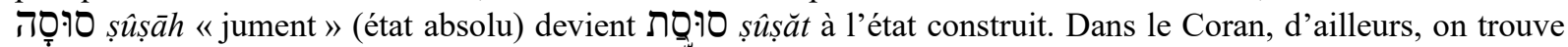

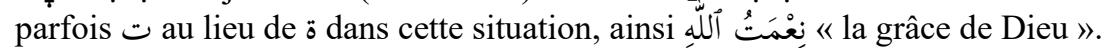

${ }^{49}$ La grammaire traditionnelle considère que les nombres de 11 à 19 sont des expressions doubles du même

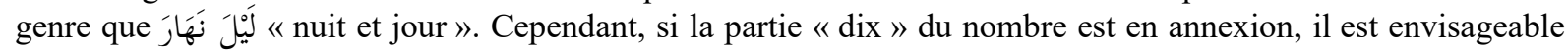
que sa finale - $a$ ne soit pas un accusatif, mais un génitif diptote. On sait en effet que les cardinaux de 3 à 10 sont diptotes quand le nombre est envisagé dans son identité, comme nom propre ; voir WRIGHT, vol. II, p. 243 C. La

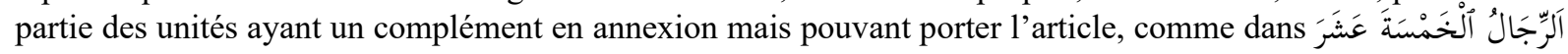
« les quinze hommes », on aurait ainsi affaire à une annexion formelle. L'annexion formelle au sens habituel ne concerne pourtant que des adjectifs (parmi lesquels les participes actifs à sens inaccompli), alors que كََّمَنَ est

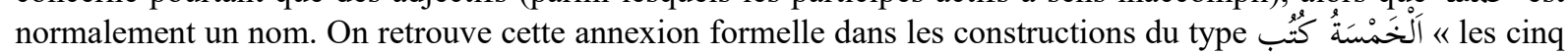
livres », qui ont acquis droit de cité depuis longtemps mais restent condamnées par certaïns puristes ; voir notamment sur ce sujet Kouloughli 2001, p. 249-251, et WRIGHT, vol. II, p. 244. 


\begin{tabular}{|c|c|}
\hline Inconstruit & Construit \\
\hline $\begin{array}{c}\text { madrasa } \\
\text { مَدْرَسَة }\end{array}$ & $\begin{array}{c}\text { madrasat } \\
\text { مَرْرَسَة }\end{array}$ \\
\hline
\end{tabular}

Bien entendu, pour إمْرَأَة imra'at, inconstruit sans article إِمرَأَ'imra'a.

\section{Conclusion}

À l'exception de la règle concernant les noms propres masculins dans l'énoncé d'une filiation, les particularités étudiées dans cette seconde partie s'intègrent parfaitement au système des états, qu'il s'agisse des situations additionnelles d'état construit ou de la déclinaison d' إمرْؤ ' et إمْرًَا. Il se confirme ainsi que le système des états constitue un excellent outil de description des déclinaisons arabes et des règles grammaticales qui les concernent; et que, vues sous cet angle, les déclinaisons arabes possèdent une structure et une cohérence remarquables.

La langue arabe apparaît ainsi comme particulièrement riche quand on la considère du point de vue des états. Même si on peut se contenter de trois états pour la décrire au niveau des déclinaisons, elle fonctionne fondamentalement sur quatre états. En outre, le fonctionnement de l'arabe par rapport aux états déterminé et construit n'est pas simple : l'état déterminé ne se résume pas en arabe au fait d'avoir l'article, puisqu'il peut aussi être requis après et ses analogues et après le لَ de négation absolue; pas plus que l'état construit - comme nous l'avons vu dans cette seconde partie - ne se réduit au fait d'avoir un complément en annexion. Le fait que les états soient presque passés sous silence à l'intérieur du monde arabisant apparaît donc comme particulièrement surprenant.

En pratique, parmi les diverses manières de décrire et de nommer les états, celle qui les oppose par paire (indéterminé / déterminé, inconstruit / construit, avec article / sans article) permet une approche graduelle des difficultés, particulièrement adaptée à l'apprentissage. Les présentations utilisant les combinaisons possibles de ces six états, quant à elles, mettent mieux en évidence l'unité de fonctionnement de toutes les déclinaisons, qu'il s'agisse de celle utilisant deux tableaux à trois états (indéterminé / déterminé sans article / avec article, pour إمرزو et إِمرَأَ ; indéterminé / emphatique / construit, pour les autres noms et adjectifs) ou de celle à quatre états valant pour tous les noms et adjectifs (indéterminé / inconstruit avec article / emphatique sans article / construit). Cette dernière, quoique étant la plus satisfaisante sur le plan théorique, est cependant sans doute trop complexe pour être utilisée en dehors d'un récapitulatif. 


\section{ANNEXE 1}

Supposons que l'on admette la construction هُمَّْ ضَارِبُو زيَيْدًا. Pour voir si est à l'état déterminé ou à l'état indéterminé, remplaçons-le par un mot sensible à cette distinction, par

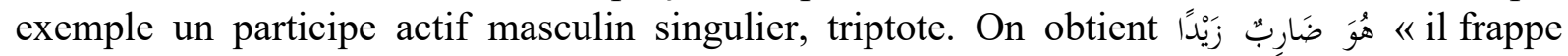
Zayd », où ضَارِب est à l'état indéterminé ; ضَارِبُو est donc aussi à l'état indéterminé. On se trouverait ici, pour les pluriels masculins externes et les duels, devant un état à la fois construit et indéterminé. A moins de bousculer la définition des états, ceci nous obligerait à ajouter un quatrième état, construit indéterminé, aux trois états élémentaires vus plus haut avant

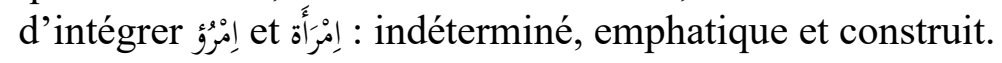

Nous montrons ici une version possible du premier tableau synoptique des déclinaisons,

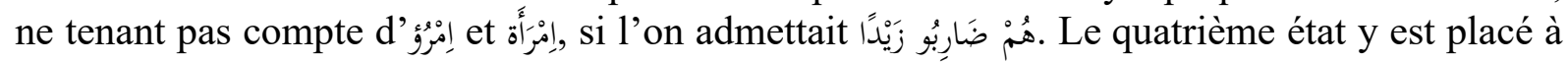
droite de l'ancien état construit, devenu état construit déterminé. Ce quatrième état aurait la bizarrerie de n'exister que pour la dernière catégorie du tableau - et uniquement pour les participes actifs, qui constitueraient ainsi une déclinaison à part. Ce qui se traduirait visuellement par le fait que le tableau synoptique, quoiqu'encore d'un seul tenant, ne serait plus rectangulaire.

Notons que l'état indéterminé se trouverait réparti entre deux sous-états : l'état indéterminé inconstruit et l'état indéterminé construit, et de ce fait constitué des deux colonnes extrêmes du tableau.

\begin{tabular}{|c|c|c|c|c|}
\hline & $\begin{array}{l}\text { Indéterminé } \\
\text { inconstruit }\end{array}$ & Emphatique & $\begin{array}{l}\text { Construit } \\
\text { déterminé }\end{array}$ & $\begin{array}{l}\text { Indéterminé } \\
\text { construit }\end{array}$ \\
\hline 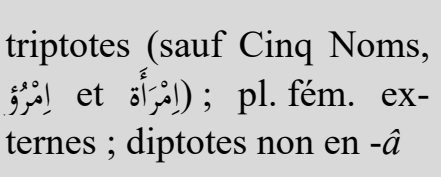 & indéterminé & \multicolumn{2}{|c|}{ déterminé } & \\
\hline Cinq Noms & indéterminé & emphatique & 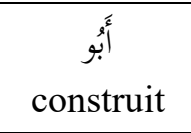 & \\
\hline $\begin{array}{l}\text { P1. masc. externes et duels } \\
\text { (sauf participes actifs) }\end{array}$ & \multicolumn{2}{|c|}{ inconstruit } & $\begin{array}{c}\text { construit } \\
\text { cóch }\end{array}$ & \\
\hline $\begin{array}{l}\text { Participes actifs pl. masc. } \\
\text { externes ou duels }\end{array}$ & \multicolumn{2}{|c|}{$\begin{array}{c}\text { ضَارِبُونَنَ } \\
\text { inconstruit }\end{array}$} & \multicolumn{2}{|c|}{$\begin{array}{c}\text { ضَارِبُو } \\
\text { construit }\end{array}$} \\
\hline
\end{tabular}

Tableau synoptique de sensibilité aux états obtenu si la situation (d) ne stipulait pas que le participe porte l'article 


\section{ANNEXE 2}

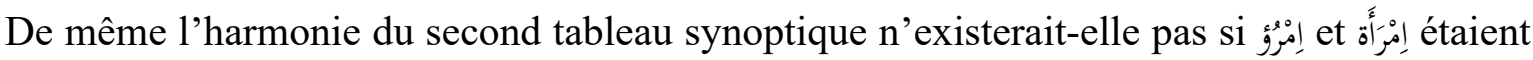
des adjectifs, ou pouvaient aussi s'utiliser comme adjectifs (notons d'ailleurs que la distinction entre nom et adjectif ne va pas de soi en arabe). En effet, il nous faudrait alors prendre en considération la possibilité que ces mots apparaissent non seulement à l'état construit sans article, mais aussi à l'état construit avec article, comme dans « la fille belle de visage ». L'état construit serait donc à remplacer par ces deux états.

Mais il ne serait pas possible de ranger les cinq états élémentaires ainsi obtenus (indéterminé, inconstruit avec article, emphatique sans article, construit sans article, construit avec article) d'une manière qui maintienne les propriétés de cohérence du tableau. On pourrait certes regrouper l'état construit sans article avec l'état emphatique sans article de façon que,

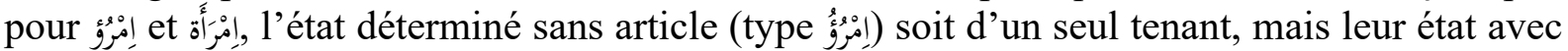

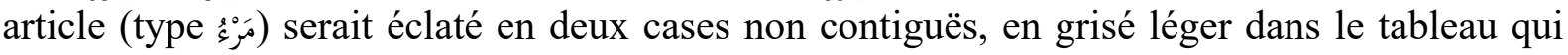
suit. En outre, un créneau apparaîtrait dans le tableau : les Cinq Noms n'ont aucune forme correspondant à l'état construit avec article, puisque ce sont des noms. Dans le tableau qui suit, nous avons d'ailleurs utilisé des adjectifs là où c'était possible, pour ne pas le surcharger. En fait, il faudrait distinguer la déclinaison des noms de celle des adjectifs, donc éclater en deux lignes les $2^{\mathrm{e}}$ et $4^{\mathrm{e}}$ lignes, ce qui ferait apparaître d'autres cases vides que celle que l'on observe dans la déclinaison des Cinq Noms. Bref, considérer إمْرَأَة et comme des adjectifs introduirait le chaos ${ }^{50}$.

\begin{tabular}{|c|c|c|c|c|c|}
\hline & Indéterminé & $\begin{array}{l}\text { Inconstruit } \\
\text { avec article }\end{array}$ & $\begin{array}{l}\text { Emphatique } \\
\text { sans article }\end{array}$ & $\begin{array}{c}\text { Construit } \\
\text { sans article }\end{array}$ & $\begin{array}{c}\text { Construit } \\
\text { avec article }\end{array}$ \\
\hline إِمرَأَة إِمْرُوُ & indéterminé & 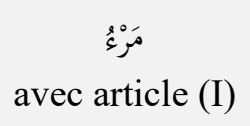 & \multicolumn{2}{|c|}{ 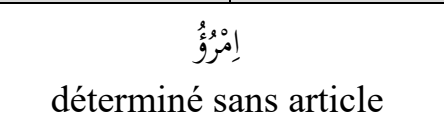 } & 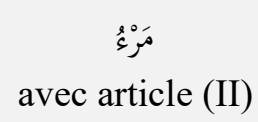 \\
\hline 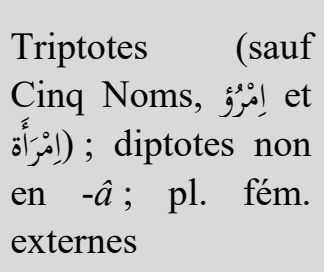 & $\begin{array}{c}\text { جَمِيلَة } \\
\text { indéterminé }\end{array}$ & \multicolumn{4}{|c|}{$\begin{array}{c}\text { جَمِيلََة } \\
\text { déterminé }\end{array}$} \\
\hline Cinq Noms & indéterminé & \multicolumn{2}{|c|}{ emphatique } & $\begin{array}{c}\text { أَبُو } \\
\text { construit }\end{array}$ & \\
\hline $\begin{array}{l}\text { Pl. masc. externes, } \\
\text { duels }\end{array}$ & \multicolumn{3}{|c|}{$\begin{array}{c}\text { جَمِيلتًَانِ } \\
\text { inconstruit }\end{array}$} & \multicolumn{2}{|c|}{$\begin{array}{c}\text { جَمِيلَتَا } \\
\text { construit }\end{array}$} \\
\hline
\end{tabular}

Tableau synoptique de sensibilité aux états obtenu si إمرئ et pouvaient être utilisés aussi comme adjectifs

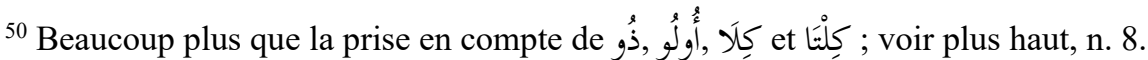




\section{BIBLIOGRAPHIE}

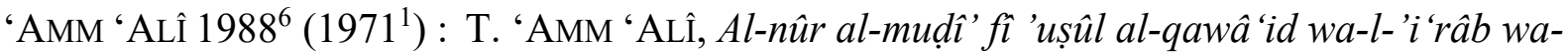
l-balâga wa-l- 'arụ̂, Damas.

BISHAI 1971 : W.B. BISHAI, Concise grammar of literary Arabic: A new approach, Dubuquet.

BlachÈre $1975\left(1978^{3}\right)$ : R. BlachÈre et M. GAUdEFroy-DEMOMBYNeS, Grammaire de l'arabe classique (morphologie et syntaxe), Paris, 1975, 1978³.

Borrmans 1967 : M. Borrmans, Grammaire d'arabe littéral. Feuilles de travail, Rome.

BrockelmANN 1941 : C. BrocKELMANN, Arabische Grammatik. Elfte, neubearbeitete Auflage der Grammatik von Socin-Brockelmann, Leipzig.

BuXTORF 1629 : J. BuXTORF, Thesaurus Grammaticus Linguae Sanctae Hebraeae, Bâle.

DiCHY 2000 : J. DiCHY, «Sur deux grammaires récentes de l'arabe moderne, observations méthodologiques », Arabica 67, p. 100-120.

FERRANDO 2000 : I. FERRANDO, «Le morphème de liaison /an/ en arabe andalou : notes de dialectologie comparée », Oriente Moderno 19 (80), 2000, 25-46.

FISCHER $1987^{2}$ (1972) : W. FISCHER, Grammatik des klassichen Arabisch, Wiesbaden 1972 , $1987^{2}$.

FLEISCH 1956 : H. FLEISCH, L'arabe classique : esquisse d'une structure linguistique, Beyrouth.

— 1961 : H. FleISCH, Traité de philologie arabe, vol. I, Beyrouth.

— 1979 : H. Fleisch, Traité de philologie arabe, vol. I, Beyrouth.

HAELEWYCK 2016 : J.-C. HAELEWYCK, Grammaire comparée des langues sémitiques. Eléments de phonétique, de morphologie et de syntaxe, Bruxelles.

JACQUESSON 2016: F. JACQUESSON, L'article, la définitude et la détermination, http://www.academia.edu/28709766/Larticle_la_définitude_et_la_détermination

JAHN 1900 : G. JAHN, Sîbawaihi's Buch über die Grammatik, übersetzt und erklärt. Zweiter Band, erster Hälfte, Berlin.

JOMIER 1983 : J. JOMIER et J. KHOUZAM, Manuel d'arabe égyptien, Paris.

KHAN 2013 : G. KHAN (éd.), Encyclopedia of Hebrew language and linguistics, Leiden / Boston.

Kouloughli 2001 : D.E. Kouloughli, « Réponses aux "Observations" de Joseph Dichy sur la grammaire de l'arabe d'aujourd'hui », Arabica 68, p. 240-253.

LANE 1863 : E.W. LANE, An Arabic-English Lexicon, Book I, Part I, London - Edinburgh.

LeCOMTE $1976^{2}$ (1968) : G. LeCOMTE, Grammaire de l'arabe, Paris, 1968.

LIPIŃSKI 1997 : E. LIPIŃSKI, Semitic Languages. Outline of a Comparative Grammar, Leuven.

MARTIN 1966 : R. MARTIN, Le mot « rien » et ses concurrents en français (du XVIe siècle à l'époque contemporaine), Paris.

RECKENDORF 1921 : H. RECKENDORF, Arabische Syntax, Heidelberg.

RETSÖ 1984-1986: J. RETSÖ, «State, Determination and Definiteness in Arabic. A reconsideration », Orientalia Suecana 33-35, p. 341-346. 
- 1997, « State and Plural Marking in Semitic », dans E. WARDINI (éd.), Built on Solid Rock. Studies in Honour of Professor Ebbe Egede Knudsen on the Occasion of his 65th Birthday April 11th 1997, Oslo, p. 268-282.

- 2010, «Status - eine vernachlässigte Kategorie der arabischen Grammatik?», in Sh. Talay et H. BobZin (éds.), Arabische Welt: Grammatik, Dichtung und Dialekte. Beiträge einer Tagung in Erlangen zu Ehren von Wolfdietrich Fischer, Wiesbaden, p. 6571.

REUCHLIN 1506 : J. REUCHLIN, De rudimentis hebraicis, Pforzheim.

SARTORI 2019 : M. SARTORI, « Definition and Determination in Medieval Arabic Grammatical Thought », dans M. Giolfo et K. Versteegh (éds.), The Foundations of Arabic Linguistics IV, Leiden / Boston, p. 253-273.

SîBAWAYHI 1970 : Le livre de Sîbawayhi, vol. II. Texte arabe publié par H. DERENBOURG, Hildesheim/New York.

Silvestre de SACY 1831a : A. I. Silvestre de SACY, Grammaire arabe, tome 1, deuxième dédition, Paris.

- 1831 b : A. I. Silvestre de SACY, Grammaire arabe, tome 2, Paris.

- 1831c : A. I. SILVESTRE de SACY, Grammaire arabe, tome 2, troisième édition, publiée par l'Institut de Carthage et revue par L. Machuel, Tunis : Société anonyme de l'imprimerie rapide, 1905.

Talloen 2007 : H. Talloen et A. Alsulaiman, 'Ayyuhā ț-tālib... Manuel d'arabe standard moderne, Antwerpen-Appeldoorn.

TIRMIDHİ 2007, English Translation of Jāmi 'AT-TIRMIDHİ. Translated by ABU KHALIYL. Volume 1, Riyadh • Jeddah • AI-Khobar • Sharjah • Lahore $\bullet$ London • Houston • New York.

Troupeau 2008 : G. Troupeau, «Réflexions sur la nature de l'i'rāb », dans J. Lentin et J. GRAND'HENRY (éds.), Moyen arabe et variétés mixtes de l'arabe à travers l'histoire, Louvain-la-Neuve : Université catholique de Louvain, Institut orientaliste, 2008, pp. 457471.

TSEREteli 1991 : K.G. TSereteli, « About the State of Nouns in Aramaic », in A. S. KAyE (éd.), Semitic Studies in honor of Wolf Leslau, vol. 2, Wiesbaden, p. 1571-1576.

VAn Der Merwe 1999 : C. H.J. VAn der Merwe, J .NAudé et .J.H. Kroeze, A Biblical Hebrew Reference Grammar, Sheffield.

VERSTEEGH 2005-2008 : K. Versteegh (éd.), Encyclopedia of Arabic Language and Linguistics, Leiden / Boston : Brill, 2005-2008.

VyCICHL 1969 : W. VyCICHL, « Der Ursprung der diptotischen Flexion im klassischen Arabischen », Le Muséon 82, p. 207-212.

Wightwick 1990 : J. Wightwick et M. GAAFAR, Mastering Arabic, Basingstoke / London.

WILMET 1985 : M. WiLMET, « A kiwi abounds in this area : note sur l'article “ indéfini générique " ", in J.-P. VAN NOPPEN et G. DEBUSSCHER (éds.), Communiquer et traduire. Hommages à Jean Dierickx, Bruxelles, p. 219-226.

Wright $1985^{4}\left(1859^{1}\right)$ : W. Wright, A grammar of the Arabic language, Cambridge. 


\title{
RÉSUMÉ
}

Cet article examine en détail la notion d'état dans le cadre des déclinaisons des noms et adjectifs de l'arabe classique. Certaines des propriétés de celles-ci peuvent se visualiser grâce aux présentations structurées des déclinaisons en fonction des cas et des états; nous montrons que ces présentations mènent à identifier un bon ordre pour les cas et pour les états. Nous établissons une définition précise des états, étudions les différentes perspectives selon lesquelles l'état peut être envisagé, exposons les avantages de son utilisation en grammaire arabe et suggérons une terminologie plus claire et plus simple que celles existantes, en mettant l'accent sur l'utilité d'une distinction nette entre les états et la définitude. Nous mettons aussi en évidence la symétrie entre les notions d'état et de cas. Tout ceci se fait, autant que possible, en lien avec les langues sémitiques en général. Nous montrons enfin que la cohérence du système formé par les cas et les états se maintient en arabe (à une exception près) lorsque l'on prend en compte les règles marginales de grammaire qui sont habituellement omises quand on envisage ce système.

\begin{abstract}
This paper performs a detailed analysis of the notion of state in the declensions of nouns and adjectives in classical Arabic. Some of the properties of declensions are easily visualized in tables using cases and states as entries. This way to describe declensions also shows that there is an optimal order for the cases and states in these tables. We propose an accurate definition of states and study the various perspectives on the subject. We show how states can be useful in Arabic grammar and suggest a simple state terminology more adapted to the current linguistic usage, stressing the necessity of a clear separation between states and definiteness. The paper also exposes the symmetry between case and state. As far as possible, all this is made keeping in mind Semitic languages in general. Last, we demonstrate that the system of cases and states keeps its coherence in Arabic (with one exception) even when some marginal grammar rules are taken into account.
\end{abstract}

